\title{
Sliding Mode Variable Impedance Control for 4RRR Redundantly Actuated Parallel Robot
}

\author{
Ya-Nan Fan \\ North China University of Technology \\ Guang-Ping He ( $\nabla$ hegp55@ncut.edu.cn ) \\ North China University of Technology \\ Xu Liang \\ North China University of Technology \\ Ting-Ting Su \\ North China University of Technology \\ Jie Zhang \\ North China University of Technology
}

Original Article

Keywords: Redundant actuation ,Compliance, Variable impedance control ,Robust stability ,Sliding mode variable impedance control

Posted Date: January 6th, 2021

DOl: https://doi.org/10.21203/rs.3.rs-139257/v1

License: (c) (1) This work is licensed under a Creative Commons Attribution 4.0 International License.

Read Full License 


\section{Title page}

\section{Sliding Mode Variable Impedance Control for 4RRR Redundantly Actuated Parallel Robot}

Ya-Nan Fan, born in 1995, is currently a master candidate at North China University of Technology, China.

Tel: 18811793292; E-mail: 535424873@qq.com

Guang-Ping He, born in 1972, is currently a professor at North China University of Technology, China. He received his PhD degree from Beihang University of China, China, in 2002. His research interests include dynamics and control of robots and micro-electromechanical devices.

E-mail: hegp55@ncut.edu.cn

Xu Liang, born in 1991, is currently a teacher at North China University of Technology, China. He received his PhD degree from The Institute of Automation, Chinese Academy of Sciences, China, in 2018. His research interests include intelligent control of rehabilitation robot and man-machine interaction.

E-mail: liangxu@ncut.edu.cn

Ting-Ting Su, born in 1991, is currently a teacher at North China University of Technology, China. She received his PhD degree from The Institute of Automation, Chinese Academy of Sciences, China, in 2018. Her research interests include trajectory planning, robotics, and intelligent control systems.

E-mail: sutingting@ncut.edu.cn

Jie Zhang, born in 1984, is currently a teacher at North China University of Technology, China. He received his PhD degree from Beihang University of China, China, in 2016. His research interests include multibody dynamics and contact mechanics.

E-mail: zhangjie@ncut.edu.cn

\section{Corresponding author: Guang-Ping He E-mail: hegp55@ncut.edu.cn}




\title{
Sliding Mode Variable Impedance Control for 4RRR Redundantly Actuated Parallel Robot
}

\author{
Ya-Nan Fan ${ }^{1}$ Guang-Ping $\mathrm{He}^{1} \cdot \mathrm{Xu} \mathrm{Liang}^{1} \cdot$ Ting-Ting Su$^{1} \cdot \mathrm{Jie} \mathrm{Zhang}^{1}$
}

Received June xx, 201x; revised February xx, 201x; accepted March xx, 201x

(c) Chinese Mechanical Engineering Society and Springer-Verlag Berlin Heidelberg 2017

\begin{abstract}
The workspace of parallel robot is limited, and the distribution characteristics of kinematics and force maneuverability are complex in the workspace. In addition, there are often operational singularities. The redundant actuation technology can not only overcome singularity problem of force maneuverability of parallel mechanism, but also eliminate the clearance of the mechanism, dynamically adjust the operating stiffness, realize the optimized operation of energy consumption, etc. In this paper, with the goal of realizing dexterous dynamic output control, the variable impedance control technology of redundantly actuated parallel robot based on operation space is studied. Compared with the traditional invariable impedance control, the variable impedance control can adapt to the change of unknown environment by time-varying controlling parameters, which provides the possibility to realize the polishing of complex curved surfaces and thin-walled parts, assembly task and improves the performance of the system and the machining quality of the parts. In order to avoid the influence of unmodeled errors and external interferences on the system, based on the sliding mode control (SMC) strategy, the variable impedance control (VIC) in the operating space of 4RRR redundantly actuated parallel robot (4RAPR) is proposed. The robust stability of the closed-loop system is analyzed and verified by Matlab programming simulation.
\end{abstract}

Keywords: Redundant actuation - Compliance - Variable impedance control - Robust stability - Sliding mode variable impedance control

Guang-Ping $\mathrm{He}$

hegp55@ncut.edu.cn

Department of Mechanical and Materials Engineering, North China University of Technology, Beijing 100144, China

\section{Introduction}

Parallel robot is a closed-loop mechanism [1] that connects moving platform with static platform through at least two independent motion chains. It has many advantages, such as large structural stiffness, high motion precision, and relatively easy acquiring the inverse motion solution, which make it one of the hot spots in current research [2]. The main limitations of parallel mechanism are small workspace, anisotropy distribution of kinematics and force operation performance in workspace, singularities and inferior maneuverability area, which bring some challenges to the global motion planning and control of robot. A feasible method for solving these issues is to use redundantly actuated technology. Redundantly actuated parallel robot means that the number of independent actuated units of the robot exceeds the number of degree of freedom (DOF) of the robot. Consequently, this kind of robot system usually generates internal load. When one or more branches of the mechanism are in singular configuration, on the one hand, redundantly actuated units can be used to achieve active stiffness control or force optimization, thereby effectively improving the global dynamic characteristics of the robot's operating space [3]. On the other hand, the generation of internal force or internal torque makes the end effector (EE) has better force operation performance [4]. Controlling the internal force by selecting appropriate 
force control algorithms can also improve the accuracy of the mechanism and adjust the operating stiffness of the robot [5]. These features that can improve the accuracy of the machine tool [6], the flexibility and force operation capability of the high-speed machine tool [7], and promote the development of human-computer interaction technology [8] have great value.

In recent years, redundantly actuated parallel robots have gradually attracted the attention of domestic and foreign scholars. For example, Chen Si [9] has studied the fault-tolerant performance of the over-constrained redundantly actuated spherical parallel mechanism, and improved the motion accuracy of parallel mechanism by removing transmission clearance. Redundantly actuated mechanism of actuation space dimension not only higher than that of configuration space dimension, also generally higher than its operating space dimension. Therefore, for specific operation space tasks, the driving force of redundantly actuated parallel mechanism has infinite sets of feasible solutions. By appropriate optimization strategies, the actuated forces can be minimized and the energy consumption can be reduced [10]. Redundantly actuated parallel mechanism has antagonistic actuation ability, which can take advantage of non-zero internal force of the mechanism to remove transmission clearance or alleviate impact vibration of the mechanism $[11,12]$. What is more attractive is that the introduction of redundant actuation provides a feasible way to eliminate the force singularity of parallel mechanism [13]. But the actuation space dimension of redundantly actuated parallel mechanism is higher than its configuration space dimension, which brings some challenges to precise dynamic modeling, inverse kinematics and dynamics analysis, and controller design of robot. Considering the errors of dynamic modeling, this paper studies the robust dynamic output control issues of redundantly actuated parallel robot based on SMC strategy. SMC is an important class of robust control method that is insensitive to the system's unmodeled errors and external disturbances, and ensures that the stability of the closed-loop system is not affected [14,15]. For the EE to implement operation tasks in contact with external environment, the commonly applied control methods are force/position hybrid control [16] and impedance control [17]. Force/position hybrid control can only be used for operating tasks where the operating force and the displacement vector are orthogonal (decoupled). While, based on the virtual concept of "mechanical impedance" and the principle of feedback equivalence, the essence of impedance control is to transform the original force adjustment target and displacement adjustment target into a second-order dynamic target system, so that a set of control inputs can achieve dynamic stability of two groups of different control tasks simultaneously. In practical applications, the EE of the robot has compliant output characteristic [18]. For practical engineering applications, the dynamic output properties of robot's EE should also be able to be adjusted, such as polishing and grinding of flexible thin-walled parts, friction welding of curved surfaces, assembly of crankshafts, etc., which is difficult to accomplish by using the invariant impedance control strategy. VIC technology is proposed for these high-level industrial application requirements, and is expected to promote the popularization and application of advanced industrial robot systems [19].

In this paper, we investigate the VIC approach for 4RAPR. The new contributions of this paper include that: (1) The proposed VIC strategy is to complete complex tasks of 4RAPR, such as polishing, grinding, assembling, and ensure the quality of parts processing. The control parameters of VIC adapt to the changes of unknown environment, as a result the EE has the characteristics of compliant output;

(2) Using Lyapunov stability conditions, the relationship between the control parameters of VIC is established, which simplifies the selection of parameters;

(3) For the purpose of enhancing the robust stability of the system, SMC strategy is combined with VIC strategy to effectively reduce the influence of unmodeled errors and external interferences. In SMC, the exponential approach term and the continuous function robust term are chosen to weaken the impact of chattering on the system.

The remainder of this paper is organized as follows: In Section 2, the dynamic model of 4RAPR is established. The 
dynamic model based on joint space contains constraint force term, which is unknown and difficult to directly measure it. So, we can try to find null space of constraint matrix to eliminate constraint force term, and then establish the dynamic model in operation space. In Section 3, VIM of 4RAPR based on operation space is proposed. Moreover, SMVIC is designed, and the stability of closed-loop system is proved by Lyapunov theory. In Section 4, Matlab programming simulation confirms the performance of VIC and SMVIC. In Section 5, conclusions and prospects.

\section{Dynamic modeling}

The establishment of dynamic model is the basis of research on robot control technology [20]. Redundantly actuated parallel robot is an over-constrained system. No matter which modeling method [21-23] is adopted, the number of effective equations will be less than the number of unknowns. As a consequence, the solution of the dynamic model is not unique, which brings certain challenges to the control technology [24]. From the perspective of system energy, the Lagrange function is employed to build dynamic model, which is convenient for the analysis of the mechanism without calculation of internal constraint reaction. Hence, in this paper, we use Lagrange function to deal with dynamic model. 4RAPR is composed of four branch chains, each of which is a two-linkage mechanism. A point $O$ represents the EE, and its Cartesian coordinate is $(x, y), A_{1}, A_{2}, A_{3}, A_{4}$ indicate positions of four motors, represented by Cartesian coordinate $\left(x_{a i}, y_{a i}\right),\left(x_{b i}, y_{b i}\right)$ is the position coordinate of the driven joint. $\theta_{i}, \varphi_{i}$ denote the active joint angle and the driven joint angle respectively. The length of active connecting rod and driven connecting rod is $a, b$ respectively, and $a=b=0.2 \mathrm{~m} .4 \mathrm{RAPR}$ is shown in Figure 1.

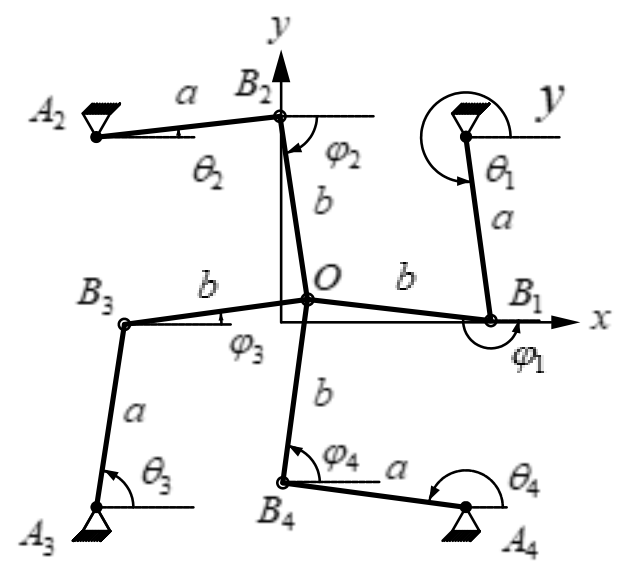

Figure 1 4RRR redundantly actuated parallel robot diagram

As can be seen in Figure 1, 4RAPR is symmetrical, thereby the mechanism can be divided into four series two-linkage mechanisms connected at one point $O$.The Lagrange function is used to establish dynamic model of four series two-linkage mechanisms, and the constraint is imposed at point $O$, and than we can obtain dynamic model of 4RAPR [25]. The series two-linkage mechanism is shown in Figure 2.

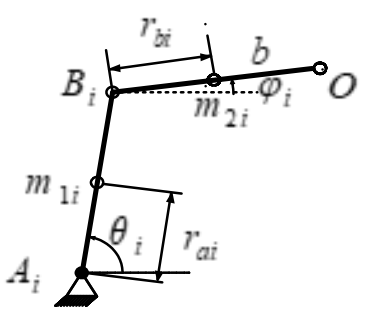

Figure 2 Mechanism diagram of two connecting linkage

The mass and the center of mass coordinate of the active rod and the driven rod of 4RAPR are $m_{a i}, m_{b i}$ and $m_{1 i}\left(x_{1 i}, y_{1 i}\right), m_{2 i}\left(x_{2 i}, y_{2 i}\right)$ respectively. $r_{a i}$ and $r_{b i}$ respectively indicate the distance from the center of mass of rod to the end of rod, where $i=1, \ldots, 4$. The calculation approach of the position of the center of mass is as follows:

$$
\begin{gathered}
\left\{\begin{array}{l}
x_{1 i}=x_{a i}+r_{a i} \cos \left(\theta_{i}\right) \\
y_{1 i}=y_{a i}+r_{a i} \sin \left(\theta_{i}\right)
\end{array}\right. \\
\left\{\begin{array}{l}
x_{2 i}=x_{a i}+a \cos \left(\theta_{i}\right)+r_{b i} \cos \left(\phi_{i}\right) \\
y_{2 i}=y_{a i}+a \sin \left(\theta_{i}\right)+r_{b i} \sin \left(\phi_{i}\right)
\end{array}\right.
\end{gathered}
$$

According to equations (1) and (2), the translational kinetic energy of the connecting rod on the horizontal plane can be expressed as: 


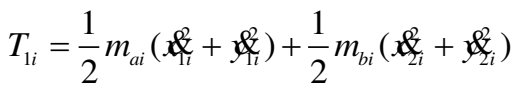

$$
\begin{aligned}
& =\frac{1}{2}\left(m_{a i} r_{a i}^{2}+m_{b i} a^{2}\right) \phi_{i}^{2}+\frac{1}{2} m_{b i} r_{a i}^{2} \phi_{i} \\
& +m_{b i} r_{b i} a \oint_{i} \phi_{i} \cos \left(\theta_{i}-\phi_{i}\right)
\end{aligned}
$$

The rotational kinetic energy of the active linkage and the driven linkage around the center of mass is calculated by:

$$
T_{2 i}=\frac{1}{6} m_{a i} r_{a i}^{2} \beta_{i}^{2}+\frac{1}{6} m_{b i} r_{b i}^{2} \phi_{i}^{2}
$$

The kinetic energy of series two-linkage is written as:

$$
T_{i}=T_{1 i}+T_{2 i}
$$

Since $4 R A P R$ is a planar mechanism, the potential energy of the mechanism is constant. Without loss of generality, this article assumes that the potential energy of planar 4RAPR is zero, such that the Lagrange function of the system satisfies $L_{i}=T_{i}$, the Lagrange function of the whole mechanism can be calculated as follows:

$$
L=L_{1}+L_{2}+L_{3}+L_{4}
$$

Using Euler-Lagrange equation [26,27]:

$$
\frac{\mathrm{d}}{\mathrm{d} t}\left(\frac{\partial L}{\partial \boldsymbol{q}}\right)-\frac{\partial L}{\partial \boldsymbol{q}}=\boldsymbol{\tau}
$$

where $\quad \boldsymbol{q}=\left[\theta_{1}, \theta_{2}, \theta_{3}, \theta_{4}, \phi_{1}, \phi_{2}, \phi_{3}, \phi_{4}\right]^{\mathrm{T}}$

$\boldsymbol{\tau}=\left[\tau_{a 1}, \tau_{a 2}, \tau_{a 3}, \tau_{a 4}, \tau_{b 1}, \tau_{b 2}, \tau_{b 3}, \tau_{b 4}\right]^{\mathrm{T}}$, the dynamic model of four series two-linkage mechanism can be obtained as follows:

$$
\text { M\& } C \&=\tau
$$

Where $\boldsymbol{M} \in R^{8 \times 8}$, is the inertia matrix in the joint space, $C \in R^{8 \times 8}$ is a coefficient matrix associated with the centrifugal and Coriolis forces in the joint space. According to the kinematic relationship of 4RAPR, the constraint equation of the closed chain mechanism can be calculated by:

$$
\boldsymbol{H}(\boldsymbol{q})=\left[\begin{array}{l}
x_{a 1}+a \cos \left(\theta_{1}\right)+b \cos \left(\phi_{1}\right) \\
-x_{a 2}-a \cos \left(\theta_{2}\right)-b \cos \left(\phi_{2}\right) \\
y_{a 1}+a \sin \left(\theta_{1}\right)+b \sin \left(\phi_{1}\right) \\
-y_{a 2}-a \sin \left(\theta_{2}\right)-b \sin \left(\phi_{2}\right) \\
x_{a 1}+a \cos \left(\theta_{1}\right)+b \cos \left(\phi_{1}\right) \\
-x_{a 3}-a \cos \left(\theta_{3}\right)-b \cos \left(\phi_{3}\right) \\
y_{a 1}+a \sin \left(\theta_{1}\right)+b \sin \left(\phi_{1}\right) \\
-y_{a 3}-a \sin \left(\theta_{3}\right)-b \sin \left(\phi_{3}\right) \\
x_{a 1}+a \cos \left(\theta_{1}\right)+b \cos \left(\phi_{1}\right) \\
-x_{a 4}-a \cos \left(\theta_{4}\right)-b \cos \left(\phi_{4}\right) \\
y_{a 1}+a \sin \left(\theta_{1}\right)+b \sin \left(\phi_{1}\right) \\
-y_{a 4}-a \sin \left(\theta_{4}\right)-b \sin \left(\phi_{4}\right)
\end{array}\right]=0
$$

Then

$$
\frac{\mathrm{d} \boldsymbol{H}}{\mathrm{d} t}=\frac{\partial \boldsymbol{H}}{\partial \boldsymbol{q}} \boldsymbol{\phi}=\boldsymbol{A}(\boldsymbol{q}) \boldsymbol{\phi}=0
$$

Where $\boldsymbol{A}(\boldsymbol{q})$ represents the set of velocity constraints and is the zero matrix of velocity vectors in the configuration space of the parallel mechanism.

Combining equations (7) with (9), we can acquire the dynamic model of 4RAPR, as shown in the following equation:

$$
\operatorname{Mat} \boldsymbol{C}=\boldsymbol{\tau}+\boldsymbol{A}^{\mathrm{T}} \lambda
$$

Where $\lambda \in R^{8}$ denotes the value of constraint force. Constraint internal force $\boldsymbol{A}^{\mathrm{T}} \boldsymbol{\lambda}$ is an internal force for the closed-loop system, that is, this term is unknown. In practical applications, the constraint term can be removed by finding the zero space of the constraint matrix. For this purpose, we define the EE position coordinate as $\boldsymbol{q}_{\mathrm{e}}=\left[\begin{array}{l}x \\ y\end{array}\right]$. The Jacobian matrix of $\boldsymbol{q}$ with respect to $\boldsymbol{q}_{\mathrm{e}}$ is $\boldsymbol{S}$, i.e., $\boldsymbol{S}=\frac{\partial \boldsymbol{q}}{\partial \boldsymbol{q}_{\mathrm{e}}}$, then

$$
\phi=S \phi_{e}
$$

Where $S \in R^{8 \times 2}$. According to equation (11), we can further get $\boldsymbol{A}(\boldsymbol{q}) \boldsymbol{\phi} \&=\boldsymbol{A}(\boldsymbol{q}) \boldsymbol{S} \boldsymbol{q}_{e}=0$, where $\boldsymbol{q}_{e}$ is the speed of the EE. When $\boldsymbol{q}_{e} \neq 0, \boldsymbol{A}(\boldsymbol{q}) \boldsymbol{S} \boldsymbol{q}_{e}=0$ always holds, so we have $\boldsymbol{A}(\boldsymbol{q}) \boldsymbol{S}=0$, i.e., $\boldsymbol{S}^{\mathrm{T}} \boldsymbol{A}(\boldsymbol{q})^{\mathrm{T}}=0$.Multiplying both sides of equation (10) by $\boldsymbol{S}^{\mathrm{T}}$, we can get the following equation:

$$
\boldsymbol{S}^{\mathrm{T}} \boldsymbol{M} \boldsymbol{S}^{\mathrm{T}} \boldsymbol{C} \&=\boldsymbol{S}^{\mathrm{T}} \boldsymbol{\tau}
$$

Applying the Jacobian matrix $\boldsymbol{S}$ and the Jacobian matrix $\boldsymbol{J}$ of the active joint vector with respect to the $\operatorname{EE} \boldsymbol{q}_{e}$, the dynamic model of 4 RAPR based on operation space can be expressed as follows:

$$
\boldsymbol{M}_{e} \boldsymbol{q}_{e}+\boldsymbol{C}_{e} \boldsymbol{q}_{e}=\boldsymbol{J}^{\mathrm{T}} \boldsymbol{\tau}_{a}
$$

Where $\boldsymbol{J} \in R^{4 \times 2}, \boldsymbol{M}_{e}=\boldsymbol{S}^{\mathrm{T}} \boldsymbol{M S}$ is inertia matrix of operation space, and $\boldsymbol{C}_{e}=\boldsymbol{S}^{\mathrm{T}}\left(\boldsymbol{M} \boldsymbol{S}^{\&} \boldsymbol{C S}\right)$ is a coefficient matrix associated with the centrifugal and Coriolis forces of operation space. Based on the above method, the joint space dynamics model (12) is equivalent to the operation space dynamics model (13). Equation (13) not only removes the unknown constraint term, reduces the scale of the system dynamics model, but also the structural characteristics of the model are consistent with the general parallel mechanism dynamic model and the open-chain mechanism dynamic model, which provides convenience for further research on the dynamic control and motion 
planning of redundantly actuated parallel robot based on operation space.

\section{Controller Design}

\subsection{Variable Impedance Control}

The essence of impedance control is to unify force control target and displacement control target in one target system where it can dynamically adjust the magnitude of motion and contact forces of EE, based on the virtual concept of "mechanical impedance" and feedback equivalence principle. For invariable impedance control, the force constraint and position constraint are steady constraints in the control task target, thereby it belongs to fixed point regulation problem. The control target of VIM is "time-varying impedance contour or trajectory". For example, mechanical parts with inconsistent thickness and stiffness at different position, when the robot is in contact with the part under the same contact forces, the deformation of the surface contact point of the part is different, and the position trajectory of the end of the robot arm will change accordingly. On the contrary, when the control accuracy of the end position is guaranteed, the contact forces at different contact points will inevitably be different, resulting in change of the processing volume. Thus, in complex environment, the dynamic characteristics of the system can be continuously changed and the control performance of the system can be improved by adjusting the control parameters of VIC.

In order to achieve the compliance of the EE, the position of the $\mathrm{EE}$ and the external forces are adjusted to reach the ideal state in real time, then the closed-loop dynamics model of VIC in operation space can be established as[28]:

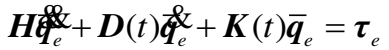

where $\boldsymbol{H}$ is positive constant matrix, $\boldsymbol{D}(t)$ and $\boldsymbol{K}(t)$ are positive time-varying matrix, $\boldsymbol{H}, \boldsymbol{D}(t), \boldsymbol{K}(t)$ are symmetric matrix, $\boldsymbol{\tau}_{e}$ is external forces. $\overline{\boldsymbol{q}}_{e}=\boldsymbol{q}_{e}-\boldsymbol{q}_{e}^{d}, \boldsymbol{q}_{e}$ is the actual position of the EE, $\boldsymbol{q}_{e}^{d}$ is the desired position of the EE.

By substituting formula (14) into (13), the control law of VIC can be written as:

$$
\left\{\begin{array}{l}
\boldsymbol{v}=\boldsymbol{H}_{e}^{-1}\left(\boldsymbol{\tau}_{e}-\boldsymbol{D}(t) \boldsymbol{\boldsymbol { q }}_{e}^{\mathrm{e}}-\boldsymbol{K}(t) \overline{\boldsymbol{q}}_{e}\right) \\
\boldsymbol{\tau}_{a}=\left(\boldsymbol{J}^{\mathrm{T}}\right)^{-1}\left(\boldsymbol{M}_{e} \boldsymbol{v}+\boldsymbol{C}_{e} \boldsymbol{\phi}_{e}^{\mathrm{t}}\right)-\left(\boldsymbol{J}^{\mathrm{T}}\right)^{-1} \boldsymbol{\tau}_{e}
\end{array}\right.
$$

Furthermore, we can prove the stability of the closed-loop system by using Lyapunov stability theory, and the detailed proof process can be found in literature [29]. The main disadvantage of the VIC closed-loop system shown in equation (15) is that it uses a simple PD controller. As we all know, PD controller is a kind of basic linear controller. The closed-loop control system based on PD controller has limited resistance to unmodeled errors and external disturbances. Subsequently, in this paper, we study the design method of SMVIC, and explore the effective method that enhances the robust stability of VIC system.

\subsection{Sliding Mode Variable Impedance Control}

Basically, SMC system is a class of discontinuous nonlinear control system which can make the system move purposefully in accordance with the desired trajectory [30], and is immune to external disturbances and parameters perturbation, etc. In practical applications, robot system usually has some uncertain factors, such as the inaccuracy of mathematical model, the interferences of the external environment and the influence of parameters perturbation, etc. By constructing a SMC law, these factors can be prevented from affecting the asymptotic stability of the closed-loop system, so as to ensure the robust stability of the closed-loop system [31].

Based on the characteristics of VIC and SMC, VIC and SMC are combined to design SMVIC. Define the sliding mode surface as:

$$
\boldsymbol{s}=\overline{\boldsymbol{q}}_{e}^{\mathrm{k}}+\Lambda \overline{\boldsymbol{q}}_{e}
$$

where $\Lambda$ is positive definite and symmetric matrix. Take the derivative of equation (16) with respect to time produces

$$
s=\boldsymbol{q}_{e}+\Lambda \underset{\boldsymbol{q}}{\mathrm{t}}=\boldsymbol{q}_{e}^{\mathrm{t}}
$$

Select the reaching law function as follows:

$$
s=-\mu \tanh (\omega s)-\lambda s
$$

Where $\mu>0, \lambda>0 .-\mu \tanh (\omega s)$ is the switching term. The introduction of the switching term can eliminate the influence of model inaccuracy and external disturbances. $-\lambda \boldsymbol{s}$ is the exponential approach term, which can ensure that the system can approach the sliding mode surface at a faster speed when the error is large. The value of $\mu$ and $\lambda$ respectively determine the rate of the system approaching the sliding mode surface $\boldsymbol{S}$ and the convergence speed of the system. The appropriate values of $\mu$ and $\lambda$ can ensure the stability of the system and make the system reach the sliding mode surface quickly in a short time. 
In the past, $-\mu$ sgns was usually used as a switching term in the approach law function to overcome the influence of disturbances and parameters perturbation, etc. However, the introduction of the switching term $-\mu$ sgns would cause chattering. Larger chattering will affect the control performance of the system, and even cause damage to the mechanical structure. Therefore, in order to alleviate the chattering in the system, the continuous function $\tanh (\omega s)$ is taken instead of the sign function sgns. Where $\omega>0$, the larger the value of $\omega$ is, the faster the change of the inflection point of $\tanh (\omega s)$ will be. When the value of $\omega$ is infinitely increased, the double tangent smooth function $\tanh (\omega s)$ is close to sgns . In order to weaken the impact of chattering and ensure robustness of the system, $\omega=5$ is taken here.

Under equations (13), (14), (17), and (18), SMVIC law can be obtained as follows:

$$
\left\{\begin{array}{l}
\boldsymbol{v}=\boldsymbol{H}_{e}^{-1}\left(\boldsymbol{\tau}_{e}-\boldsymbol{D}(t) \boldsymbol{\boldsymbol { q }}_{e}^{\mathrm{U}}-\boldsymbol{K}(t) \overline{\boldsymbol{q}_{e}}\right) \\
\boldsymbol{q}_{e}=\frac{1}{\Lambda}\left(-\mu \tanh (\omega \boldsymbol{s})-\lambda \boldsymbol{s}-\boldsymbol{v}+\boldsymbol{q}_{e}\right. \\
\boldsymbol{\tau}_{a}=\left(\boldsymbol{J}^{\mathrm{T}}\right)^{-1}\left(\boldsymbol{M}_{e} \boldsymbol{v}+\boldsymbol{C}_{e} \boldsymbol{q}_{e}^{\mathrm{\psi}}-\boldsymbol{\tau}_{e}\right)
\end{array}\right.
$$

Select the Lyapunov candidate function as:

$$
\mathbf{V}=\frac{1}{2} \boldsymbol{s}^{\mathrm{T}} \boldsymbol{s}+\frac{1}{2} \overline{\boldsymbol{q}}_{e}^{\mathrm{T}} \boldsymbol{\beta}(t) \overline{\boldsymbol{q}}_{e}
$$

where $\boldsymbol{\beta}(t)$ is the undetermined time-varying matrix.

Proposition1: Consider the dynamic model of 4RAPR based on operation space (13) and the target VIC closed-loop dynamic system (14), if the stiffness matrix $\boldsymbol{K}(t)$ is a continuous function and $\sigma>0$ exists, which makes $K^{\&}(t) \leq \sigma$, inequality group satisfies

$$
\left\{\begin{array}{l}
\Lambda>0 \\
\boldsymbol{H}^{-1} \boldsymbol{K}(t)+\boldsymbol{H}^{-1} \boldsymbol{D}(t) \Lambda-\Lambda^{2}>0 \\
-\boldsymbol{H}^{-1} \boldsymbol{D}(t)+\Lambda<0 \\
\frac{1}{2} \boldsymbol{H}^{-1} \boldsymbol{K}^{\&}(t)+\frac{1}{2} \boldsymbol{H}^{-1} \boldsymbol{D}(t) \Lambda-\Lambda \boldsymbol{H}^{-1} \boldsymbol{K}(t)<0
\end{array}\right.
$$

Then the closed-loop system is globally asymptotically stable at $\boldsymbol{\tau}_{e}=0$.

Proof: Let $\boldsymbol{\tau}_{e}=0$, then

$$
\begin{aligned}
& \boldsymbol{V}^{\&}=\boldsymbol{s}^{\mathrm{T}} \boldsymbol{s}+\overline{\boldsymbol{q}}_{e}^{\mathrm{T}} \boldsymbol{\beta}(t) \boldsymbol{\boldsymbol { q }}_{e}^{\mathrm{E}}+\frac{1}{2} \overline{\boldsymbol{q}}_{e}^{\mathrm{T}} \boldsymbol{\beta}(t) \overline{\boldsymbol{q}}_{e}
\end{aligned}
$$

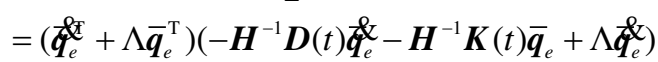

$$
\begin{aligned}
& +\overline{\boldsymbol{q}}_{e}^{\mathrm{T}} \boldsymbol{\beta}(t) \overline{\boldsymbol{q}}_{e}^{\mathrm{k}}+\frac{1}{2} \overline{\boldsymbol{q}}_{e}^{\mathrm{T}} \boldsymbol{\beta}(t) \overline{\boldsymbol{q}}_{e}
\end{aligned}
$$

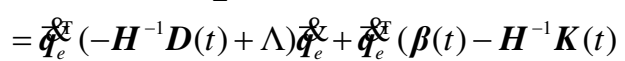

$$
\begin{aligned}
& \left.-\boldsymbol{H}^{-1} \boldsymbol{D}(t) \Lambda+\Lambda^{2}\right) \overline{\boldsymbol{q}}_{e}+\overline{\boldsymbol{q}}_{e}^{\mathrm{T}}\left(\frac{1}{2} \boldsymbol{\beta}^{\&}(t)-\Lambda \boldsymbol{H}^{-1} \boldsymbol{K}(t)\right) \overline{\boldsymbol{q}}_{e}
\end{aligned}
$$

For the sake of removing the non-quadratic term of equation (21) and ensuring the positive definiteness of the Lyapunov candidate function, let $\boldsymbol{\beta}(t)=\boldsymbol{H}^{-1} \boldsymbol{K}(t)+\boldsymbol{H}^{-1} \boldsymbol{D}(t) \Lambda-\Lambda^{2}>0$,by deriving it, we can get $\boldsymbol{\beta}(t)=\boldsymbol{H}^{-1} \boldsymbol{K}(t)+\boldsymbol{H}^{-1} \boldsymbol{H}(t) \Lambda$, then the formula (21) can be rewritten as:

$$
\begin{aligned}
\boldsymbol{W}= & =\boldsymbol{\boldsymbol { q }}_{e}^{\ll}\left(-\boldsymbol{H}^{-1} \boldsymbol{D}(t)+\Lambda\right) \boldsymbol{\boldsymbol { q }}_{e}^{\mathrm{e}}+\overline{\boldsymbol{q}}_{e}^{\mathrm{T}}\left(\frac{1}{2} \boldsymbol{H}^{-1} \boldsymbol{K}^{\&}(t)\right. \\
& \left.+\frac{1}{2} \boldsymbol{H}^{-1} \boldsymbol{H}(t) \Lambda-\Lambda \boldsymbol{H}^{-1} \boldsymbol{K}(t)\right) \overline{\boldsymbol{q}}_{e}
\end{aligned}
$$

To ensure $\boldsymbol{W}_{<}<\mathbf{0}$, the inequality group must be satisfied

$$
\left\{\begin{array}{l}
-\boldsymbol{H}^{-1} \boldsymbol{D}(t)+\Lambda<0 \\
\frac{1}{2} \boldsymbol{H}^{-1} \boldsymbol{K}^{\&}(t)+\frac{1}{2} \boldsymbol{H}^{-1} \boldsymbol{B}(t) \Lambda \\
-\Lambda \boldsymbol{H}^{-1} \boldsymbol{K}(t)<0
\end{array}\right.
$$

Equation (23) can be further simplified as:

$$
\left\{\begin{array}{l}
\Lambda \boldsymbol{H}<\boldsymbol{D}(t) \\
\boldsymbol{D} \&(t)<-\frac{1}{\Lambda} \boldsymbol{K}^{\&}(t)+2 \boldsymbol{K}(t)
\end{array}\right.
$$

In equation (24), $\boldsymbol{K}^{\&}(t)$ is bounded, i.e., $\boldsymbol{K}^{\&}(t) \leq \sigma, \sigma \geq 0$. There are always $\Lambda$ and $\boldsymbol{\rho}=\left[\begin{array}{l}\rho_{1} \\ \rho_{2}\end{array}\right], \rho_{1}$ and $\rho_{2}$ are both positive constants that satisfy $-\frac{1}{\Lambda} \boldsymbol{K}^{\&}(t)+2 \boldsymbol{K}(t)>\boldsymbol{\rho}$, obviously, $\boldsymbol{D}(t)=\boldsymbol{H} \Lambda+\boldsymbol{\rho}$ holds.

Based on the above analysis, the set of inequalities (23) guarantee that $\boldsymbol{\psi}_{\text {is }}$ negative definite, and because $\mathbf{V}$ is positive definite, when $\overline{\boldsymbol{q}}_{e} \rightarrow \infty$, $\boldsymbol{q}_{e}^{k} \rightarrow \infty, \mathbf{V} \rightarrow \infty$ is unbounded. Moreover, when reaching the sliding mode surface $s=0, \quad \&=0$, that is to say, $t \rightarrow \infty$, 
$\overline{\boldsymbol{q}}_{e} \rightarrow 0, \quad \boldsymbol{q}_{e}^{\mathrm{K}} \rightarrow 0$, thereby the closed-loop system is globally asymptotically stable. The stability condition (20) of the closed-loop system (14) can be proved.

Remark1: Inequalities $\quad \Lambda>0 \quad$ and $\boldsymbol{\beta}(t)=\boldsymbol{H}^{-1} \boldsymbol{K}(t)+\boldsymbol{H}^{-1} \boldsymbol{D}(t) \Lambda-\Lambda^{2}>0$ guarantee the positive definiteness of the Lyapunov function. Meanwhile, the parameter $\Lambda>0$ satisfies the Hurwitz condition in the SMC. The damping parameter $\boldsymbol{D}(t)=\boldsymbol{H} \Lambda+\boldsymbol{\rho}$ builds the relationship between the control parameters and simplifies the selection of parameters. The stiffness parameters $\boldsymbol{K}(t)$ should be selected according to the specific operation task, at the same time, $\boldsymbol{K}^{\&}(t)$ should be bounded, which brings some limitations to the selection of parameters.

Remark2: Indeed, VIC and SMVIC designed in this paper only include position feedback. If we need to obtain the velocity, acceleration and torque signals of the EE and avoid increasing the complexity of the structure by installing the velocity sensor, the observer can be designed. In addition, in order to improve the force tracking capability of EE, the force feedback control loop should be further added.

\subsection{Robust asymptotic stability analysis for closed-loop system}

Generally speaking, the physical model of 4RAPR (13), owing to structure size error, parameters perturbation, external disturbances, etc., is imprecision. Considering the above factors, we can define the dynamic model as:

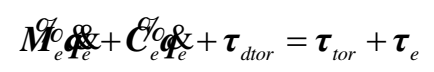

where $\boldsymbol{\tau}_{\text {tor }}=\boldsymbol{J}^{\mathrm{T}} \boldsymbol{\tau}_{a}, \boldsymbol{M}_{e}^{O}=\boldsymbol{M}_{e}-\Delta \boldsymbol{M}_{e}, \mathscr{C}_{e}^{O}=\boldsymbol{C}_{e}-\Delta \boldsymbol{C}_{e} \cdot \boldsymbol{M}_{e}^{O}$ and

$\mathrm{C}_{e}$ respectively represent the actual value of inertia matrix and a coefficient matrix associated with the centrifugal and Coriolis forces based on operation space. $\Delta \boldsymbol{M}_{e}$ and $\Delta \boldsymbol{C}_{e}$ respectively indicate the error of inertia matrix and a coefficient matrix associated with the centrifugal and Coriolis forces based on operation space. $\boldsymbol{\tau}_{d t o r}$ are external disturbances.

Substituting formula (25) into (17) yelds

$$
\begin{aligned}
& s b=\mathbb{q}_{e}^{2}+\Lambda \mathbb{q}_{e}^{\mathrm{t}} \\
& =\Psi_{e}+\Lambda \mathbb{q}_{e}^{e} \\
& =\boldsymbol{M}_{e}^{\sigma^{1}}\left(\boldsymbol{\tau}_{\text {tor }}+\boldsymbol{\tau}_{e}-\boldsymbol{\tau}_{d t o r}-\boldsymbol{C}_{e}^{o} \boldsymbol{q}_{e}\right)-\boldsymbol{q}_{e}+\Lambda \boldsymbol{q}_{e}^{e}
\end{aligned}
$$

Proposition2: In order to make the system have robust asymptotic stability, the control law is taken as:

$$
\begin{aligned}
& \boldsymbol{\tau}_{\text {tor }}+\boldsymbol{\tau}_{e}=M_{e}+\boldsymbol{C}_{e} \boldsymbol{q}_{e}-k_{i}^{2 / s} \\
& -k_{p} \tanh (\omega \boldsymbol{s})-\boldsymbol{M}_{e} \Lambda \boldsymbol{q}_{e}^{\mathrm{x}}-\boldsymbol{C}_{e} \Lambda \overline{\boldsymbol{q}}_{e}
\end{aligned}
$$

where $k_{i}^{\%}$ and $k_{p}^{\%}$ are constants and $k_{i}^{\%}>0, k_{p}^{\%}>0$.

Proof: Take the Lyapunov candidate function as:

$$
\mathbf{V}=\frac{1}{2} \boldsymbol{s}^{\mathrm{T}} \boldsymbol{M}_{e} \boldsymbol{s}
$$

Differential Lyapunov candidate function satisfies

$$
\boldsymbol{Z}=\boldsymbol{s}^{\mathrm{T}} \boldsymbol{M}_{e}^{0} \boldsymbol{s} \&+\frac{1}{2} \boldsymbol{s}^{\mathrm{T}} \boldsymbol{M}_{e} \boldsymbol{s}
$$

According to the properties of the dynamic model, $\mathscr{M}_{e}-2 \boldsymbol{C}_{e}$ is skew symmetric matrix, therefore equation (29) can be further denoted as:

$$
\begin{aligned}
& \boldsymbol{Z}=\boldsymbol{s}^{\mathrm{T}} \mathscr{M}_{e}^{0} \boldsymbol{s}+\frac{1}{2} \boldsymbol{s}^{\mathrm{T}} \stackrel{\&}{\boldsymbol{H}_{e}} \boldsymbol{s} \\
& =\boldsymbol{s}^{\mathrm{T}} \boldsymbol{M}_{e} \boldsymbol{s} \&+\frac{1}{2} \boldsymbol{s}^{\mathrm{T}}\left(\boldsymbol{M}_{e}-2 \boldsymbol{C}_{e}\right) \boldsymbol{s}+\boldsymbol{s}^{\mathrm{T}} \boldsymbol{C}_{e} \boldsymbol{s} \\
& =\boldsymbol{s}^{\mathrm{T}} \boldsymbol{M}_{e}{ }_{e} \boldsymbol{s}+\boldsymbol{s}^{\mathrm{T}} \boldsymbol{C}_{e} \boldsymbol{s}
\end{aligned}
$$

Under equation (16), (26), and (30), we can get

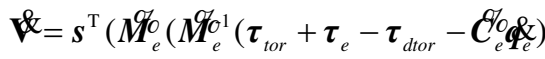

$$
\begin{aligned}
& \left.\left.-\boldsymbol{q}_{e}+\Lambda \overline{\boldsymbol{q}}_{e}^{\mathrm{k}}\right)+\boldsymbol{C}_{e}\left(\overline{\boldsymbol{q}}_{e}^{\mathrm{E}}+\Lambda \overline{\boldsymbol{q}}_{e}\right)\right) \\
& =\boldsymbol{s}^{\mathrm{T}}\left(\boldsymbol{\tau}_{t o r}+\boldsymbol{\tau}_{e}-\boldsymbol{\tau}_{d t o r}+\left(\boldsymbol{M}_{e}-\Delta \boldsymbol{M}_{e}\right)\left(\Lambda \boldsymbol{\boldsymbol { q }}_{e}^{\mathrm{e}}-\boldsymbol{C}_{e}\right)\right. \\
& \left.-\boldsymbol{C}_{e} \boldsymbol{\Psi}_{e}+\Delta \boldsymbol{C}_{e} \boldsymbol{q}_{e}+\left(\boldsymbol{C}_{e}-\Delta \boldsymbol{C}_{e}\right)\left(\overline{\boldsymbol{q}}_{e}^{\mathrm{k}}+\Lambda \overline{\boldsymbol{q}}_{e}\right)\right) \\
& =\boldsymbol{s}^{\mathrm{T}}\left(\boldsymbol{\tau}_{t o r}+\boldsymbol{\tau}_{e}+\boldsymbol{M}_{e} \Lambda \boldsymbol{q}_{e}^{\mathrm{Z}}-M_{e}-C_{e} \boldsymbol{\psi}_{e}^{\mathrm{\Sigma}}\right. \\
& +\boldsymbol{C}_{e} \hat{\boldsymbol{q}}_{e}^{\mathrm{\&}}+\boldsymbol{C}_{e} \Lambda \overline{\boldsymbol{q}}_{e}-\boldsymbol{\tau}_{d t o r}-\Delta \boldsymbol{M}_{e} \Lambda \boldsymbol{\boldsymbol { q }}_{e}^{\mathrm{E}} \\
& \left.+\Delta \boldsymbol{M}_{e} \boldsymbol{l}_{e}+\Delta \boldsymbol{C}_{e} \boldsymbol{q}_{e}^{\ll}-\Delta \boldsymbol{C}_{e} \overline{\boldsymbol{q}}_{e}^{\mathrm{K}}-\Delta \boldsymbol{C}_{e} \Lambda \overline{\boldsymbol{q}}_{e}\right)
\end{aligned}
$$

In equation (31), let

$$
\begin{aligned}
& \boldsymbol{\Phi}^{0}=\boldsymbol{\tau}_{d t o r}+\Delta \boldsymbol{M}_{e} \Lambda \boldsymbol{q}_{e}^{\mathrm{E}}-\Delta \boldsymbol{M}_{e} \\
& -\Delta \boldsymbol{C}_{e} \boldsymbol{q}_{e}^{\gtrless}+\Delta \boldsymbol{C}_{e} \boldsymbol{q}_{e}^{\gtrless}+\Delta \boldsymbol{C}_{e} \Lambda \overline{\boldsymbol{q}}_{e}
\end{aligned}
$$

be the model parameters error and external disturbances. 
Invoking equation (27), equation (31) can be further simplified as:

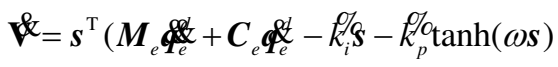

$$
\begin{aligned}
& -\boldsymbol{M}_{e} \Lambda \overline{\boldsymbol{q}}_{e}^{\mathrm{j}}-\boldsymbol{C}_{e} \Lambda \overline{\boldsymbol{q}}_{e}+\boldsymbol{M}_{e} \Lambda \boldsymbol{q}_{e}^{\mathrm{j}}-\boldsymbol{M}_{e} \\
& -\boldsymbol{C}_{e} \boldsymbol{\phi}+\boldsymbol{C}_{e} \dot{\boldsymbol{q}}+\boldsymbol{C}_{e} \Lambda \overline{\boldsymbol{q}}_{e}-\boldsymbol{\phi} \bar{q} \\
& =s^{\mathrm{T}}\left(-k_{i} / \mathrm{s}-k_{p}^{/} \tanh (\omega \boldsymbol{s})-\boldsymbol{\Phi}\right)
\end{aligned}
$$

Because of the double tangent function $\tanh (\omega s)=\frac{\mathrm{e}^{\omega s}-\mathrm{e}^{-\omega s}}{\mathrm{e}^{\omega s}+\mathrm{e}^{-\omega s}}[32]$, for any sliding mode surface $s$, when $\omega>0$, there is always

$$
\begin{aligned}
& s \tanh (\omega s)=|s \tanh (\omega s)| \\
& =|s||\tanh (\omega s)| \approx|s||\operatorname{sgn}(s)| \geq 0
\end{aligned}
$$

Suppose $\quad \mid \not \phi \leq Q^{c} \quad$ and $\quad k_{p}^{\%}>Q_{c} \quad, \quad$ one has

$$
\boldsymbol{k}=-\boldsymbol{s}^{\mathrm{T}} \boldsymbol{k}_{i}^{/} \boldsymbol{s}-k_{p} \boldsymbol{s}^{\mathrm{T}} \tanh (\omega \boldsymbol{s})-\boldsymbol{s}^{\mathrm{T}} \boldsymbol{\Phi}^{0}<0
$$

when

$\overline{\boldsymbol{q}}_{e} \rightarrow \infty, \quad \overline{\boldsymbol{q}}_{e}^{\mathrm{e}} \rightarrow \infty, \mathbf{V} \rightarrow \infty$ is unbounded. Therefore, for the system (13) including unmodeled errors and external disturbances, the closed-loop system is globally robust asymptotically stable under the SMVIC (19)[33]. Proposition 2 is proved.

Remark3: In order to reduce the chattering of the closed-loop system, this paper uses a continuous function $\tanh (\omega s)$ to approximate the discontinuous sign function $\operatorname{sgn}(s)$, so the value of $k_{p}^{\%}$ should be strictly larger than the value of $\&$ to ensure the global asymptotic stability of the system. The value of $k_{p}^{/ 0}$ is affected by the size of modeling uncertainties and external disturbances. When modeling uncertainties and external disturbances are larger, $k_{p}^{\%}$ should also be increased to make sure that the system has strong robustness [34].

\section{MATLAB Simulation}

For VIC method and SMVIC method of 4RAPR, a model is built in MATLAB's simulink for simulation analysis, where chap2_1ctrl is VIC or SMVIC, and chap2_1plant is the controlled objective. The simulink model diagram is shown in Figure 3.

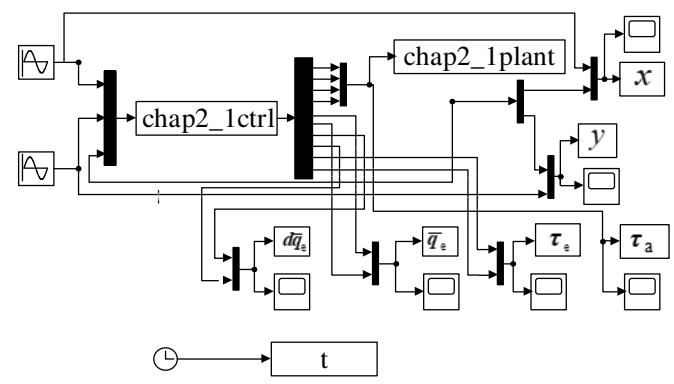

Figure 3 Simulink model diagram

In the simulation process of VIC, the control parameter $\boldsymbol{H}$ can not be arbitrarily selected, and should be adjusted within a certain range according to the actual inertial matrix parameter, in order to maintain the stability of the system. In this paper, $\boldsymbol{K}(t)$ is chosen arbitrarily. In practical applications, it should be determined according to specific operation tasks. The values of specific parameters controlled for VIC are shown in Table 1.

Table 1 Variable impedance control parameters

\begin{tabular}{lccc}
\hline \multicolumn{1}{c}{ Parameters } & symbols & value & unit \\
\hline Desired position & $\boldsymbol{q}_{e}^{d}$ & {$\left[\begin{array}{l}0.03 \cos (t) \\
0.03 \sin (t)\end{array}\right]$} & $\mathrm{m}$ \\
Initial position & $\boldsymbol{q}_{e}$ & {$\left[\begin{array}{l}0 \\
0\end{array}\right]$} & $\mathrm{m}$ \\
Initial velocity & $\boldsymbol{\Phi}_{e}$ & {$\left[\begin{array}{l}0 \\
0\end{array}\right]$} & $\mathrm{m} / \mathrm{s}$ \\
Desired inertia matrix & $\boldsymbol{H}$ & {$\left[\begin{array}{ll}0.08 & 0 \\
0 & 0.1\end{array}\right]$} & $\mathrm{Kg}$ \\
Desired damping matrix & $\boldsymbol{D}(t)$ & {$\left[\begin{array}{ll}1.6 & 0 \\
0 & 2\end{array}\right]$} & $\mathrm{N} / \mathrm{s}$ \\
Desired stiffness matrix & $\boldsymbol{K}(t)$ & $\left.\begin{array}{cc}7 \sin (2 t)+22 & 0 \\
0 & 5 \cos (2 t)+18\end{array}\right]$ & $\mathrm{N} / \mathrm{m}$ \\
External force & & {$\left[\begin{array}{c}0.2 \\
0\end{array}\right]$} & $\mathrm{N}$ \\
\hline
\end{tabular}

Figure 4-7 show the simulation results of VIC. Figure 4 and 5 denote the position errors and velocity errors of the EE. Because the stiffness is time-varying, the closed-loop system has to adjust the robot's end position to guarantee 
the stability of the interaction forces of the EE, so that the position error curves are fluctuating. Figure 6 shows the four drive torques of the parallel mechanism. It can be seen that the introduction of redundant actuation makes the distribution of drive torques more even. Figure 7 shows the interaction forces. From the curves, it can be seen that the interaction forces can be stabilized at desired value. According to the dynamic relationship between force and position of VIC, VIC can not only adjust the position, but also adjust the stiffness of the system through the given contact external forces, so as to realize the compliance control and make sure the safety of the processing objective and the robot system.

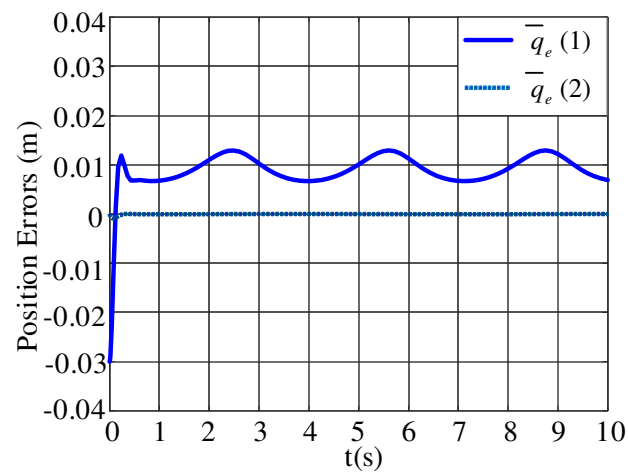

Figure 4 Position errors trajectory of EE

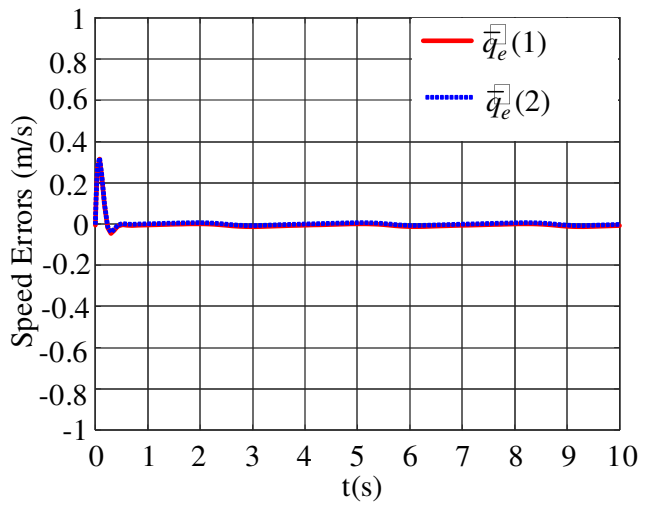

Figure 5 Speed errors trajectory of EE

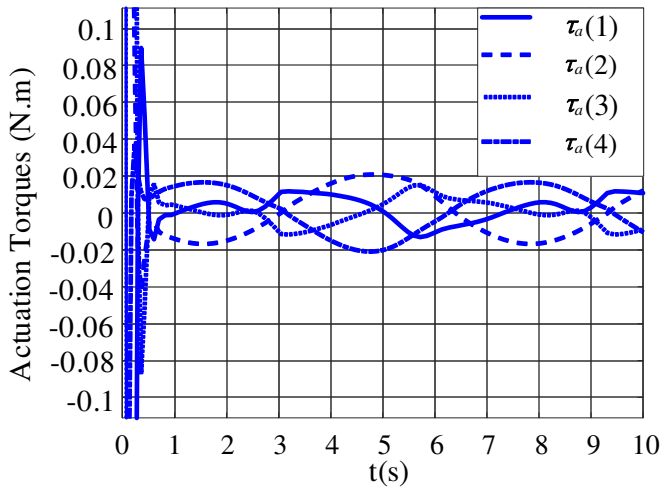

Figure 6 Actuated moment trajectory of each actuated unit

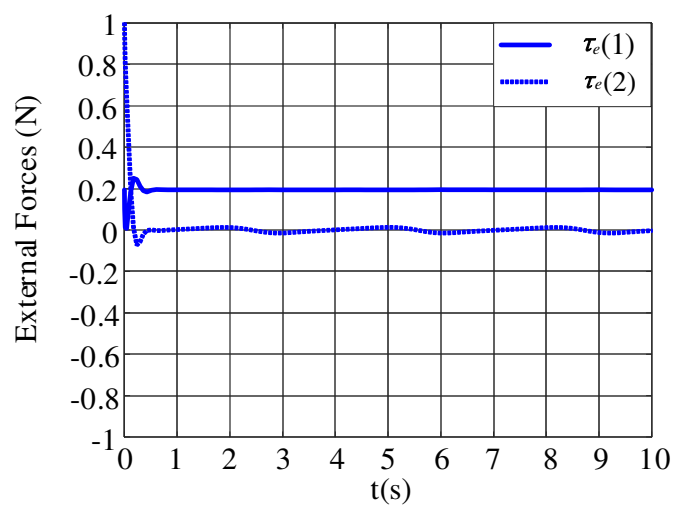

Figure 7 Interaction forces

In the simulation of SMVIC, the desired position, initial position, initial velocity and impedance control parameters of the EE are the same as those adopted in the simulation of VIC. The parameters of SMC are shown in Table 2, where $\boldsymbol{d}(t)$ are the unmodeled errors and external disturbance errors.

Table 2 Sliding mode variable impedance control parameters

\begin{tabular}{cccccc}
\hline Symbols & $\Lambda$ & $k_{p}^{\%}$ & $k_{i}^{/ c}$ & $\omega$ & $\boldsymbol{d}(t)$ \\
\hline Value & 10 & 1.1 & 10 & 5 & {$\left[\begin{array}{c}\sin (2 t) \\
0\end{array}\right]$} \\
\hline
\end{tabular}

The simulation results are shown in Figure 8-11. Figure 8 and 9 respectively show the position error curves and velocity error curves of the EE. It can be clearly seen from the curves that the state of the controlled system is stable. Figure 10 shows the driving torques. Due to external disturbances and unmodeled errors, the driving torques of the parallel mechanism are larger than that of VIC. Because the external disturbances imposed on the 
controlled objective are time-varying, although the interaction forces in Figure 11 fluctuate, it tends to a given value within a certain period of time.

Comparing VIC with SMVIC, it can be seen that the errors of two different closed-loop systems shown in Figure.4, 5, 8, 9 are stable. In light of the characteristics of PD controller, it is difficult for VIC to stabilize the desired contact forces in the case of unmodeled errors and external disturbances. Presuming that the system is not affected by unmodeled errors and external interferences, as shown in Figure 11, VIC can be stabilized to the desired contact forces, which confirms the correctness of theoretical analysis. However, the actual operating environment is often relatively complex, thus VIC is not easily applied in engineering practice. Through theoretical analysis of SMVIC and simulation results of the contact external forces in Figure 11, it can be seen that the design of SMVIC provides the possibility and reference value for practical engineering applications.

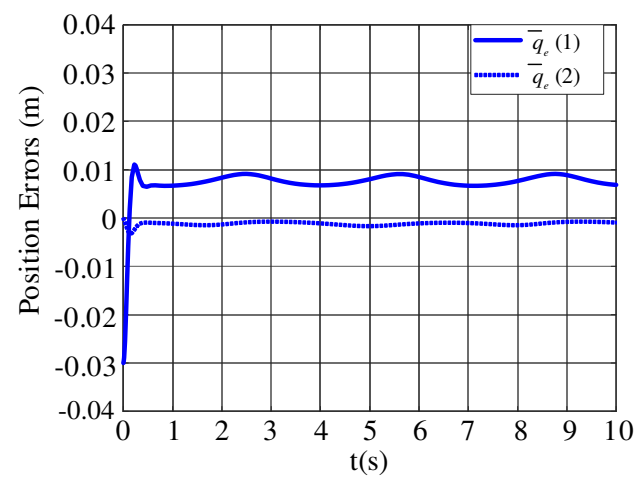

Figure 8 Position errors trajectory of EE

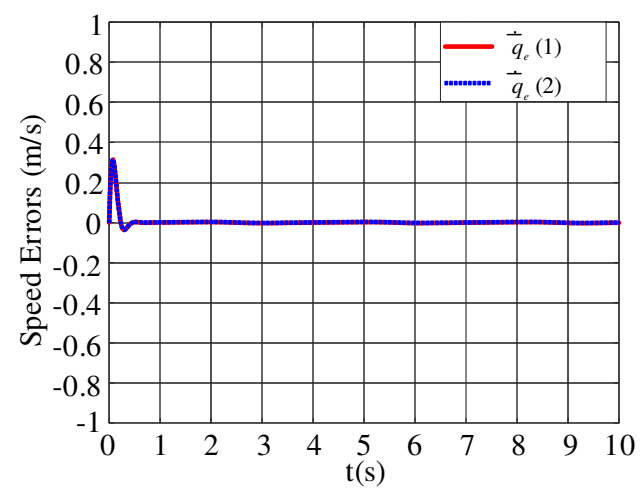

Figure 9 Speed errors trajectory of EE

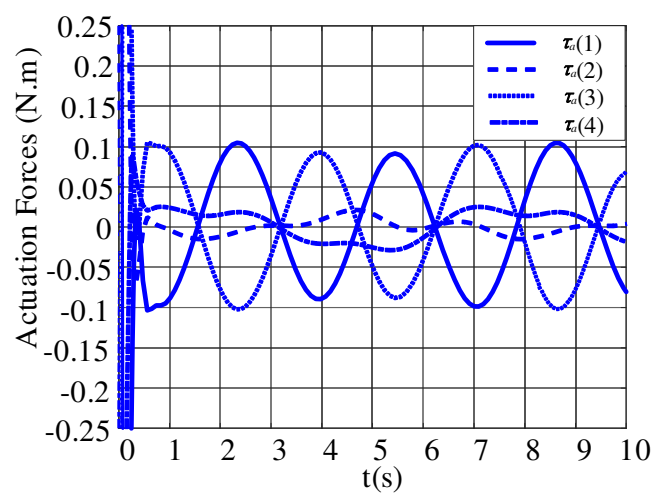

Figure 10 Actuated moment trajectory of each actuated unit

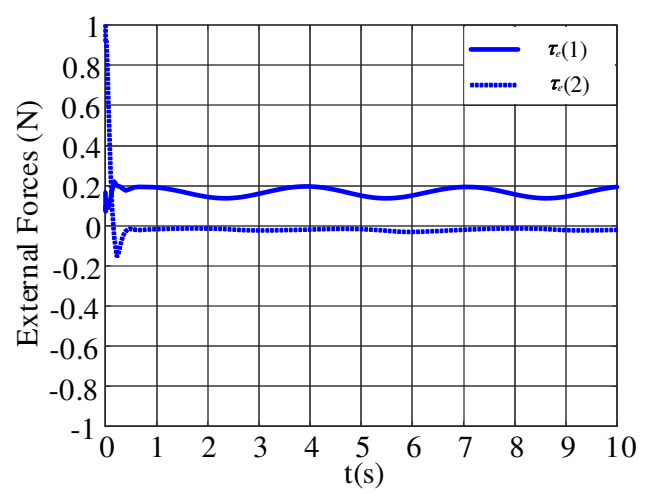

Figure 11 Interaction forces

\section{Conclusions and Prospects}

In this paper, VIC and SMVIC are designed for 4RAPR to achieve compliant control and enhance the robust stability of the system. By adjusting the dynamic relationship between force and position, effectively improve the robot's compliance, safety, and interactive operation performance. The stability conditions of Lyapunov function are used to establish the relationship SMVIC parameters, which simplifies the selection of parameters. Through MATLAB simulation analysis, we found that SMVIC can effectively restrain the influence of uncertainties such as external disturbances and unmodeled errors, and make interaction forces and trajectory tracking errors asymptotically stable. But the SMVIC will increase the energy consumption under the condition of external interferences. During simulation process, VIC parameters and SMVIC parameters are determined based on experience. In future research work, appropriate optimization algorithms can be selected to optimize VIC 
parameters and SMVIC parameters, so as to reduce energy consumption of the system as far as possible and improve the performance of trajectory tracking. In unknown and complex operating environment, such as the polishing and grinding of flexible thin-wall parts, friction welding of curved surfaces, etc., how to design control parameter $\boldsymbol{K}(t)$ still requires further research.

\section{Declaration}

\section{Acknowledgements}

The authors sincerely thanks to Professor Guang-Ping He of North China University of Technology for his critical discussion and reading during manuscript preparation.

\section{Funding}

Supported by National Key Research and Development Plan of China (Grant No. 2019YFB1309600), National Natural Science Foundation of China (Grant No. 51775002), Beijing Natural Science Foundation (Grant No. KZ202010009015).

\section{Availability of data and materials}

The datasets supporting the conclusions of this article are included within the article.

\section{Authors' contributions}

The author' contributions are as follows: Guang-Ping He was in charge of the whole trial; Ya-Nan Fan wrote the manuscript; Xu Liang, Ting-Ting Su, Jie Zhang assisted with sampling and laboratory analyses.

\section{Competing interests}

The authors declare no competing financial interests.

\section{Consent for publication}

Not applicable

\section{Ethics approval and consent to participate}

Not applicable

\section{References}

[1] V Garg, S B Nokleby, J A Carretero. Wrench capability analysis of redundantly actuated spatial parallel manipulators. Mechanism and Machine Theory. 2009, 44(5): 1070-1081.

[2] J Kim, F C Park, S J Ryu, et al. Design and analysis of a redundantly actuated parallel mechanism for rapid machining.
IEEE Transactions on robotics and automation, 2001, 17(4): 423-434.

[3] S Kock, W Schumacher. A parallel x-y manipulator with actuation redundancy for high-speed and active-stiffness applications. International Conference on Robotics and Automation, Leuven Belgium, 1998: 2295-2300.

[4] C Gosselin, L Schreiber. Redundancy in Parallel Mechanisms: A Review. Applied Mechanics Reviews, 2018, 70(1).

[5] T Harada, M Nagase. Impedance control of a redundantly actuated 3-DOF planar parallel link mechanism using direct drive linear motors. 2010 IEEE International Conference on Robotics and Biomimetics, Tianjin China: IEEE, 2010: 501-506.

[6] L Wang, J Wu, J Wang, et al. An experimental study of a redundantly actuated parallel manipulator for a 5-DOF hybrid machine tool. IEEE-ASME Transactions on Mechatronics, 2009, 14(1): 72-81.

[7] S B Nokleby, R Fisher, R P Podhorodeski, et al. Force capabilities of redundantly-actuated parallel manipulators. Mechanism and machine theory, 2005, 40(5): 578-599.

[8] D Chakarov. Study of the antagonistic stiffness of parallel manipulators with actuation redundancy. Mechanism and Machine Theory, 2004, 39(6): 583-601.

[9] S Chen, S W Fan. Study on approach to enhance precision fault tolerance ability for spherical parallel mechanisms based on over-constrained redundancy actuation. China Mechanical Engineering, 2012, 23(16):1985-1990.

[10] G Lee, S K Sul, J Kim. Energy-saving method of parallel mechanism by redundant actuation. International Journal of Precision Engineering and Manufacturing-Green Technology, 2015, 2(4): 345-351.

[11] G P He, X L Tan, X H Zhang, et al. Optimal synthesis of a planar 3-DOF overactuated full-compliant parallel mechanism. Robot, 2006, 28(6):623-628,635.

[12] A Muller. Internal preload control of redundantly actuated parallel manipulators-its application to backlash avoiding control. IEEE Transactions on Robotics and Automation, 2005, 21(4): 668-677.

[13] J Kim, J C Hwang, J S Kim, et al. Eclipse-II: a new parallel mechanism enabling continuous 360-degree spinning plus three-axis translational motions. International Conference on Robotics and Automation. Seoul, Korea: IEEE, 2001: 3274-3280.

[14] G Q Gao, J Wen, X J Liu, et al. Synchronous smooth sliding mode control for parallel mechanism based on coupling analysis. 
International Journal of Advanced Robotic Systems, 2013, 10(173): $1-10$.

[15] H Cheng, G F Liu, Y K Yiu, et al. Advantages and dynamics of parallel manipulators with redundant actuation. Proceedings 2001 IEEE/RSJ International Conference on Intelligent Robots and Systems. IEEE, 2001, 1: 171-176.

[16] T Yoshikawa, T Sugie, M Tanaka. Dynamic hybrid position/force control of robot manipulators--controller design and experiment. International Conference on Robotics and Automation. Japan: IEEE, 1987: 699-705.

[17] $\mathrm{N}$ Hogan. Impedance control: an approach to manipulation. American Control Conference. USA, 1984: 304-313.

[18] F Caccavale, B Siciliano, L Villani. The Tricept robot: dynamics and impedance control. IEEE/ASME transactions on mechatronics, 2003, 8(2): 263-268.

[19] D W Franklin, R Osu, E Burdet, et al. Adaptation to stable and unstable dynamics achieved by combined impedance control and inverse dynamics model. Journal of neurophysiology, 2003, 90(5): 3270-3282.

[20] D Constantinescu, I Chau, S P Dimaio, et al. Haptic rendering of planar rigid-body motion using a redundant parallel mechanism. International Conference on Robotics and Automation, San, Francisco: IEEE, 2000: 2440-2445.

[21] R D Gregorio, V Parenticastelli. Dynamics of a class of parallel wrists. Journal of Mechanical Design, 2004, 126(3): 436-441.

[22] A Fattah, G Kasaei. Kinematics and dynamics of a parallel manipulator with a new architecture. Robotica, 2000, 18(5): $535-543$.

[23] M J Liu, C X Li, C N Li, et al. Dynamics analysis of the Gough-Stewart platform manipulator. IEEE Transactions on Robotics and Automation, 2000, 16(1): 94-98.

[24] Y Jiang, T Li, L Wang, et al. The dynamic modeling, redundant-force optimization and dynamic performance analyses of a parallel kinematic machine with actuation redundancy. Robotica, 2015, 33(2): 241-263.

[25] X Niu, G Gao, X Liu, et al. Dynamics modeling and experiments of 3-DOF parallel mechanism with actuation redundancy. Transactions of the Chinese Society of Agricultural Engineering, 2013, 29(16): 31-41.

[26] F H Ghorbel, O Chetelat, R Gunawardana, et al. Modeling and set point control of closed-chain mechanisms: Theory and experiment. IEEE Transactions on control systems technology, 2000, 8(5), 801-815.

[27] H Cheng, Y K Yiu, Z Li. Dynamics and control of redundantly actuated parallel manipulators. IEEE/ASME Transactions on Mechatronics, 2003, 8(4), 483-491.

[28] K Kronander, A Billard. Stability considerations for variable impedance control. IEEE Transactions on Robotics, 2016, 32(5): 1298-1305.

[29] G P He, Y N Fan, T T Su, et al. Variable impedance control of cable actuated continuum manipulators. International Journal of Control, Automation and Systems, 2020, 18(7): 1-14.

[30] J K Liu. Sliding Mode Control Design and MATLAB Simulation (The Third)The Basic Theory and Design Method. Beijing: Tsinghua University Press, 2012.

[31] Z M Chen, Z Y Wang, J G Zhang. Sliding Mode Variable Structure Control Theory and Application. Beijing: Publishing House of Electronics Industry, 2012

[32] M P Aghababa, M E Akbari. A chattering-free robust adaptive sliding mode controller for synchronization of two different chaotic systems with unknown uncertainties and external disturbances. Applied Mathematics and Computation, 2012, 218(9): 5757-5768.

[33] R M Murray, Z Li, S S Sastry, et al. A mathematical introduction to robotic manipulation. CRC press, 1994.

[34] H Lee, V I Utkin. Chattering suppression methods in sliding mode control systems. Annual reviews in control, 2007, 31(2): 179-188.

\section{Biographical notes}

Ya-Nan Fan, born in 1995, is currently a master candidate at North China University of Technology, China.

Tel: 18811793292; E-mail: 535424873@qq.com

Guang-Ping He, born in 1972, is currently a professor at North China University of Technology, China. He received his $\mathrm{PhD}$ degree from Beihang University of China, China, in 2002. His research interests include dynamics and control of robots and micro-electromechanical devices.

E-mail: hegp55@ncut.edu.cn

Xu Liang, born in 1991, is currently a teacher at North China University of Technology, China. He received his $\mathrm{PhD}$ degree from The Institute of Automation, Chinese Academy of Sciences, China, in 2018. His research interests include intelligent control of rehabilitation robot and man-machine interaction.

E-mail: liangxu@ncut.edu.cn

Ting-Ting Su, born in 1991, is currently a teacher at North China University of Technology, China. She received his $\mathrm{PhD}$ degree from The Institute of Automation, Chinese Academy of Sciences, 
China, in 2018. Her research interests include trajectory planning, robotics, and intelligent control systems.

E-mail: sutingting@ncut.edu.cn

Jie Zhang, born in 1984, is currently a teacher at North China University of Technology, China. He received his $\mathrm{PhD}$ degree from Beihang University of China, China, in 2016. His research interests include multibody dynamics and contact mechanics.

E-mail: zhangjie@ncut.edu.cn 
Figures

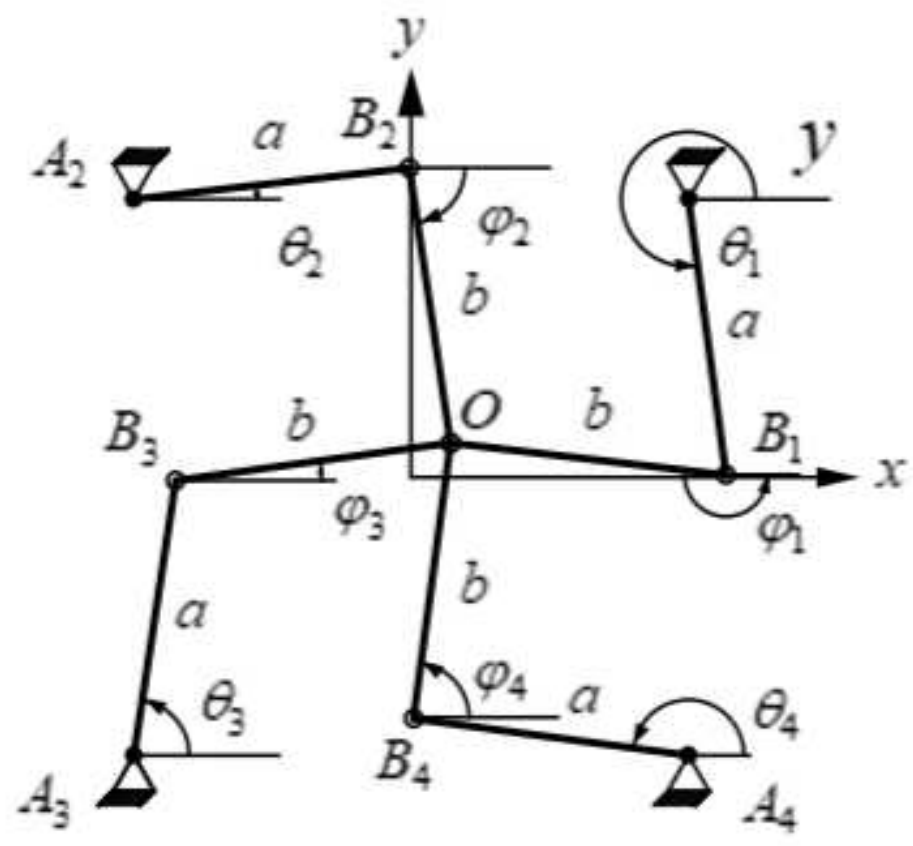

Figure 1

4RRR redundantly actuated parallel robot diagram

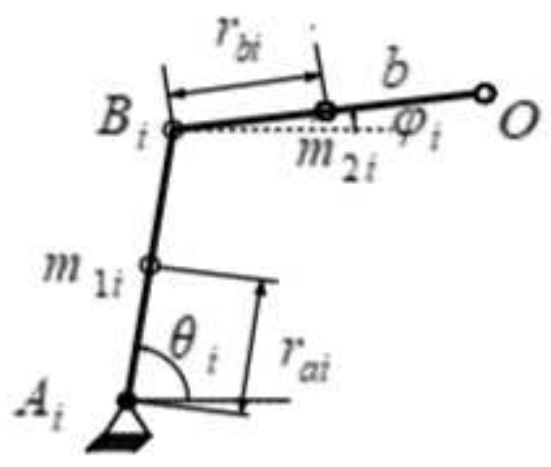

Figure 2

Mechanism diagram of two connecting linkage 


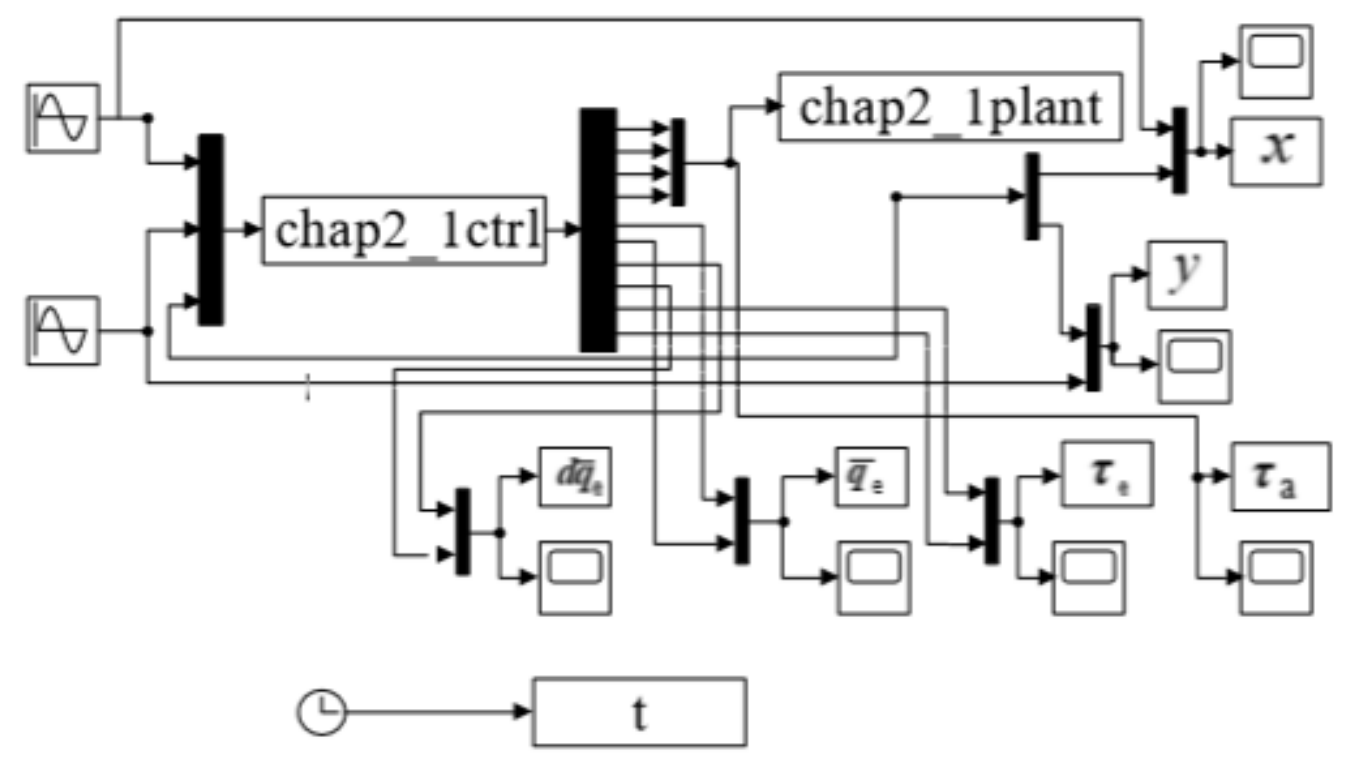

Figure 3

Simulink model diagram

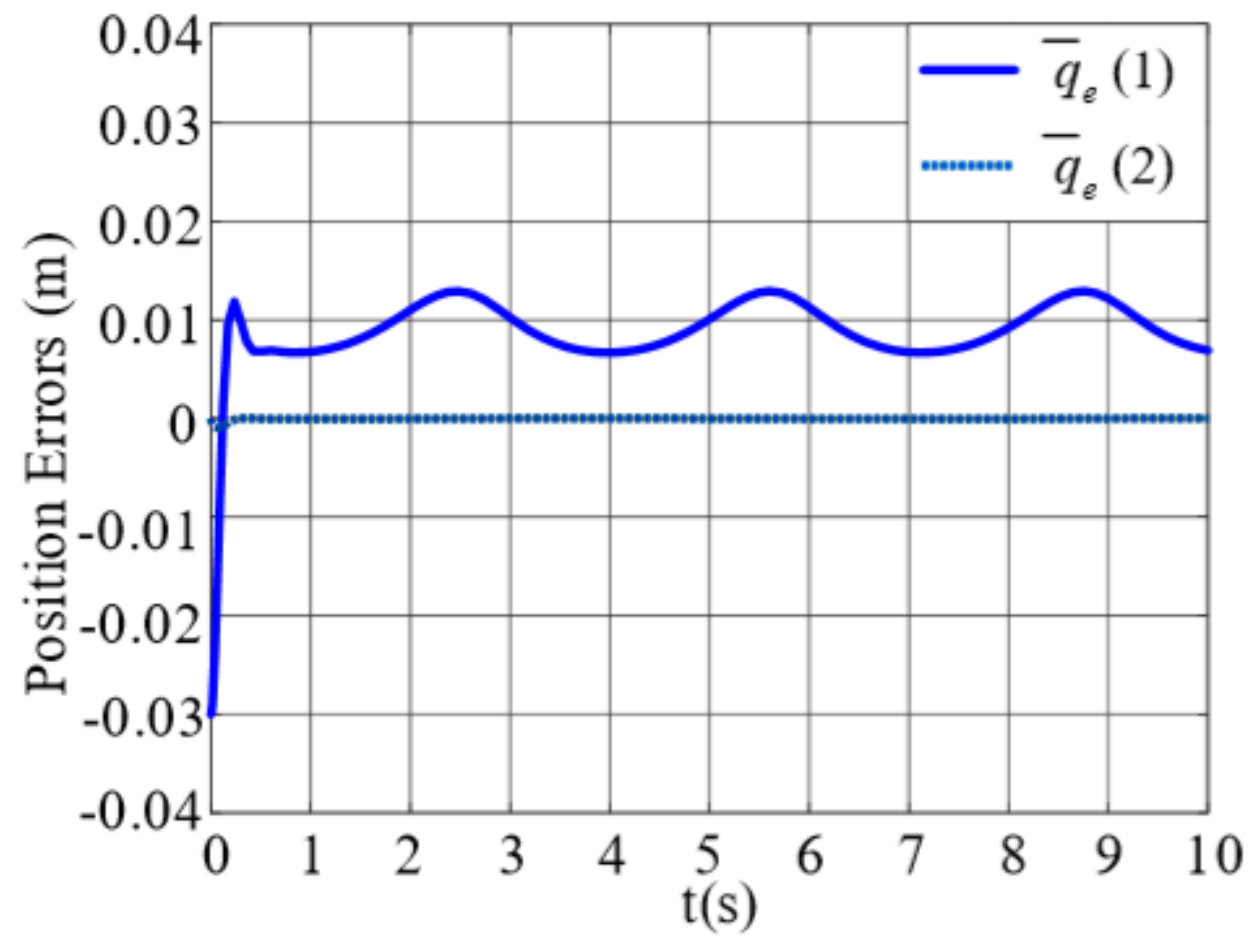

Figure 4

Position errors trajectory of EE 


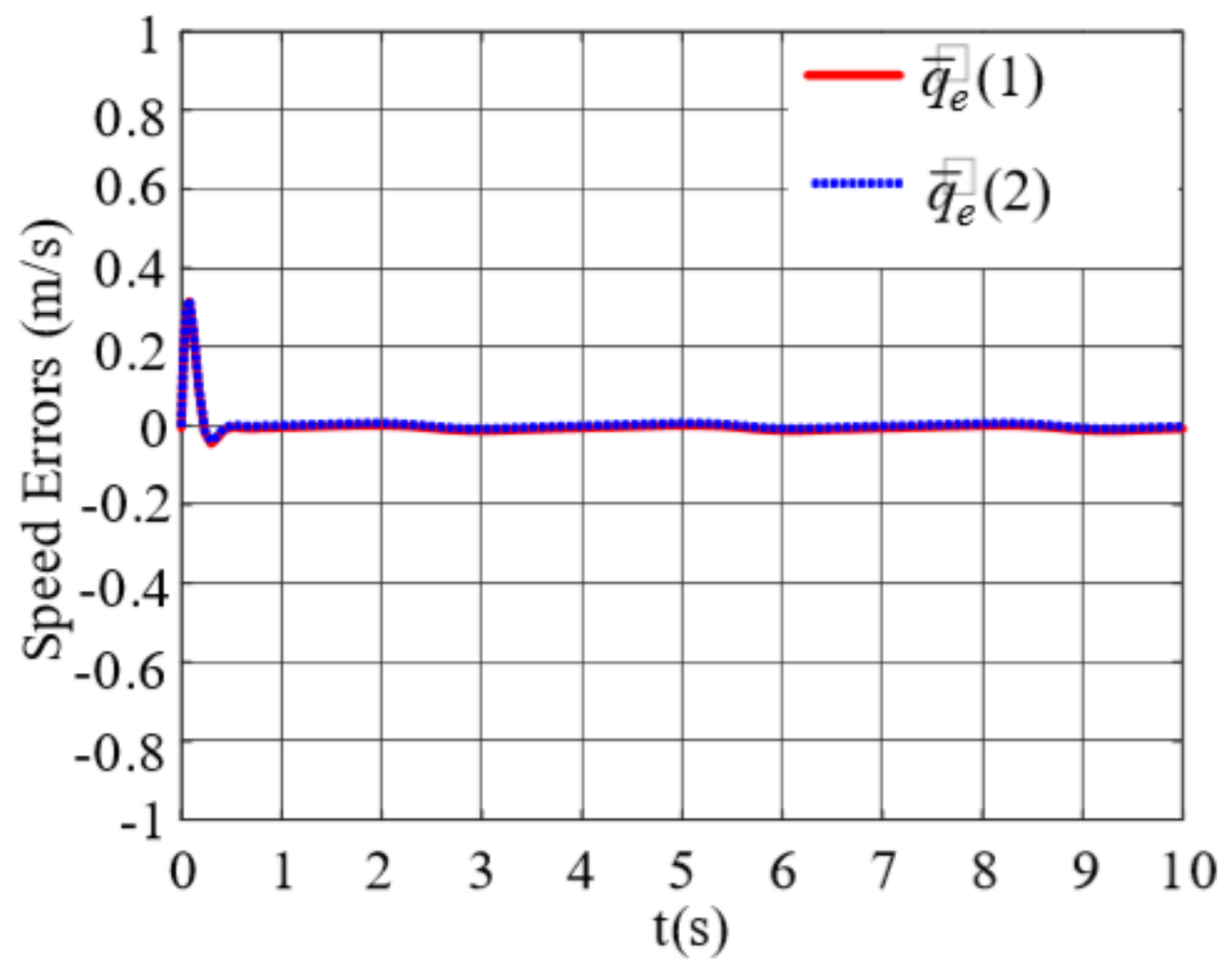

Figure 5

Speed errors trajectory of EE

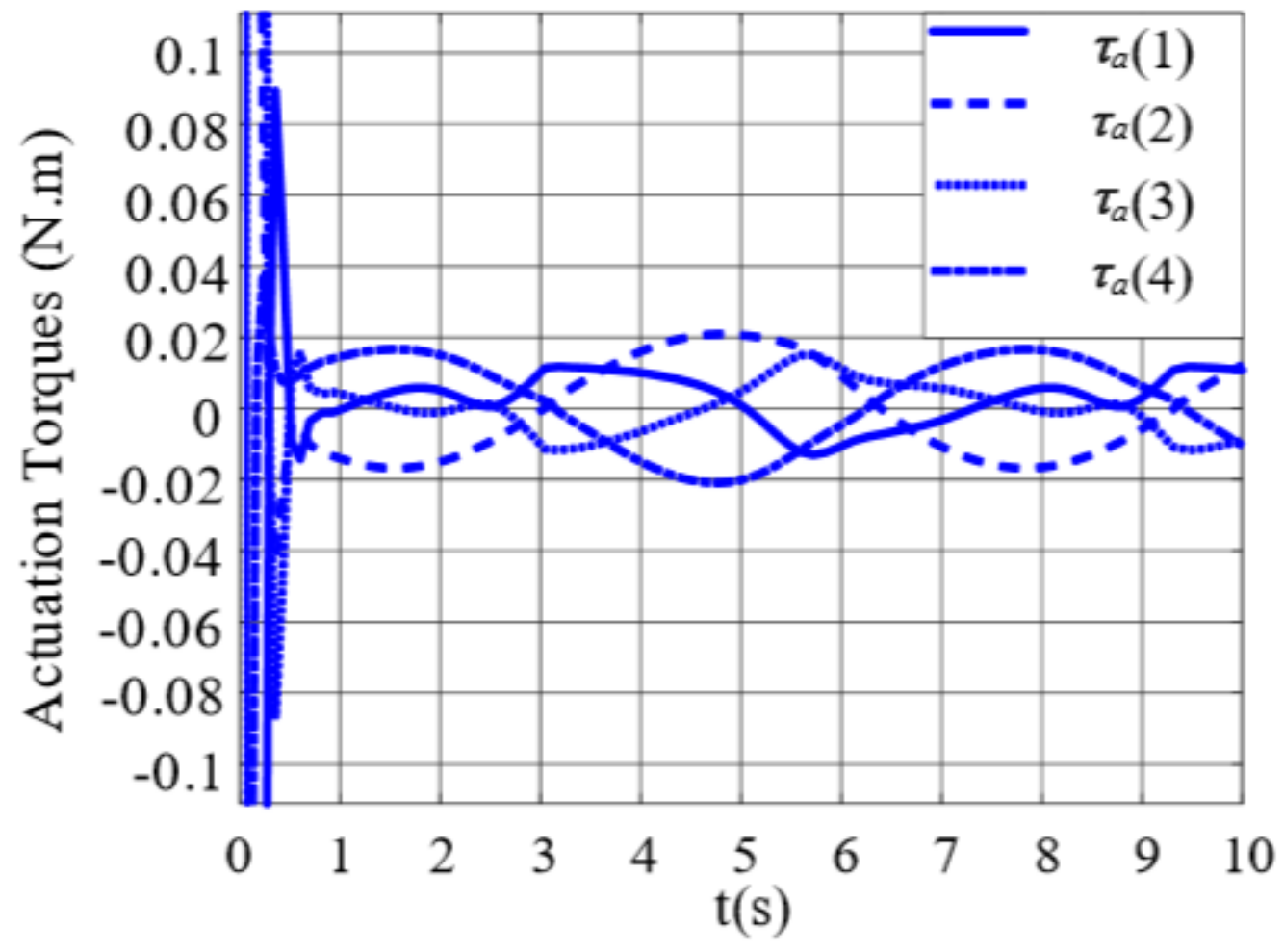

Figure 6 
Actuated moment trajectory of each actuated unit

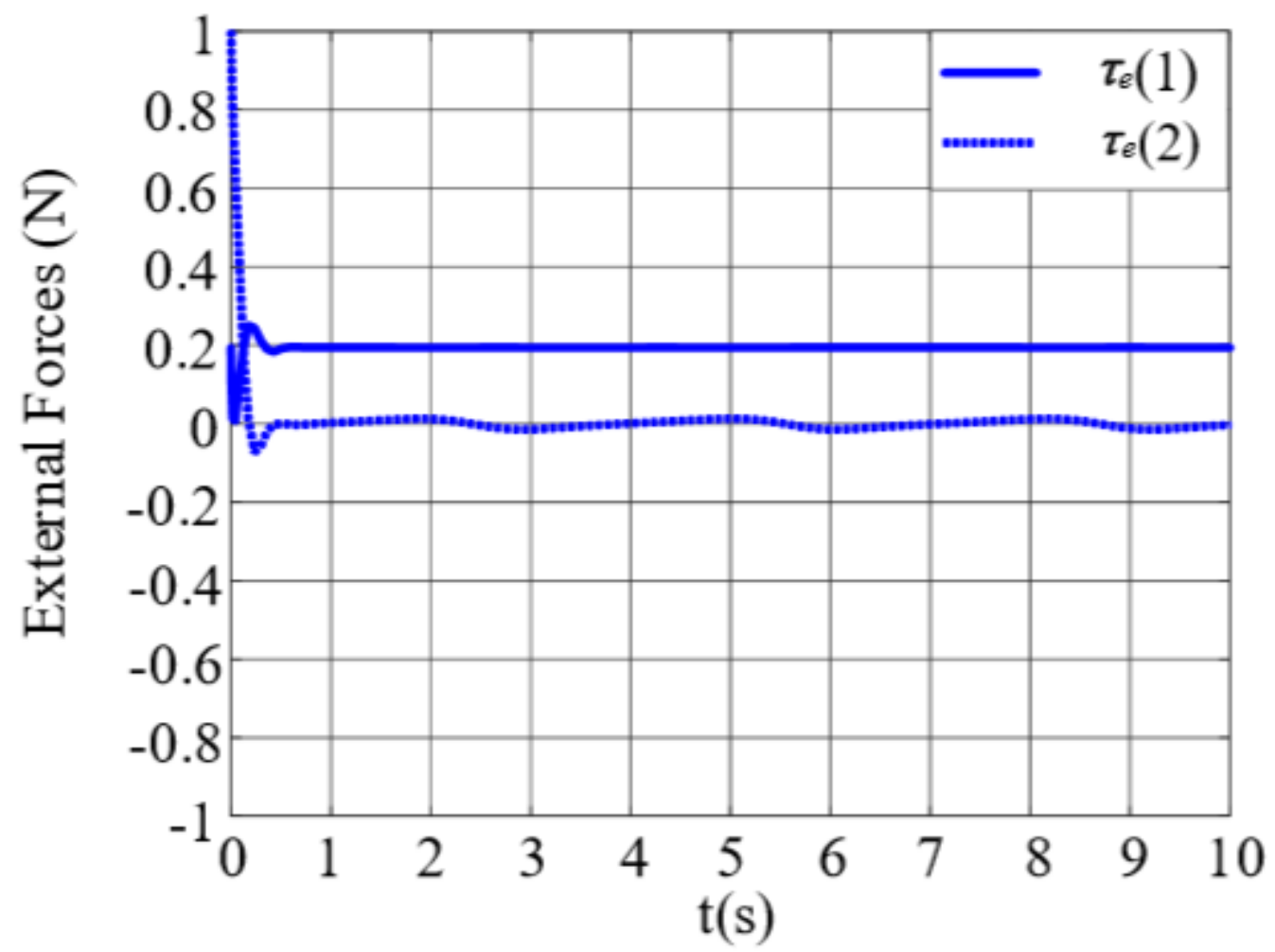

Figure 7

Interaction forces

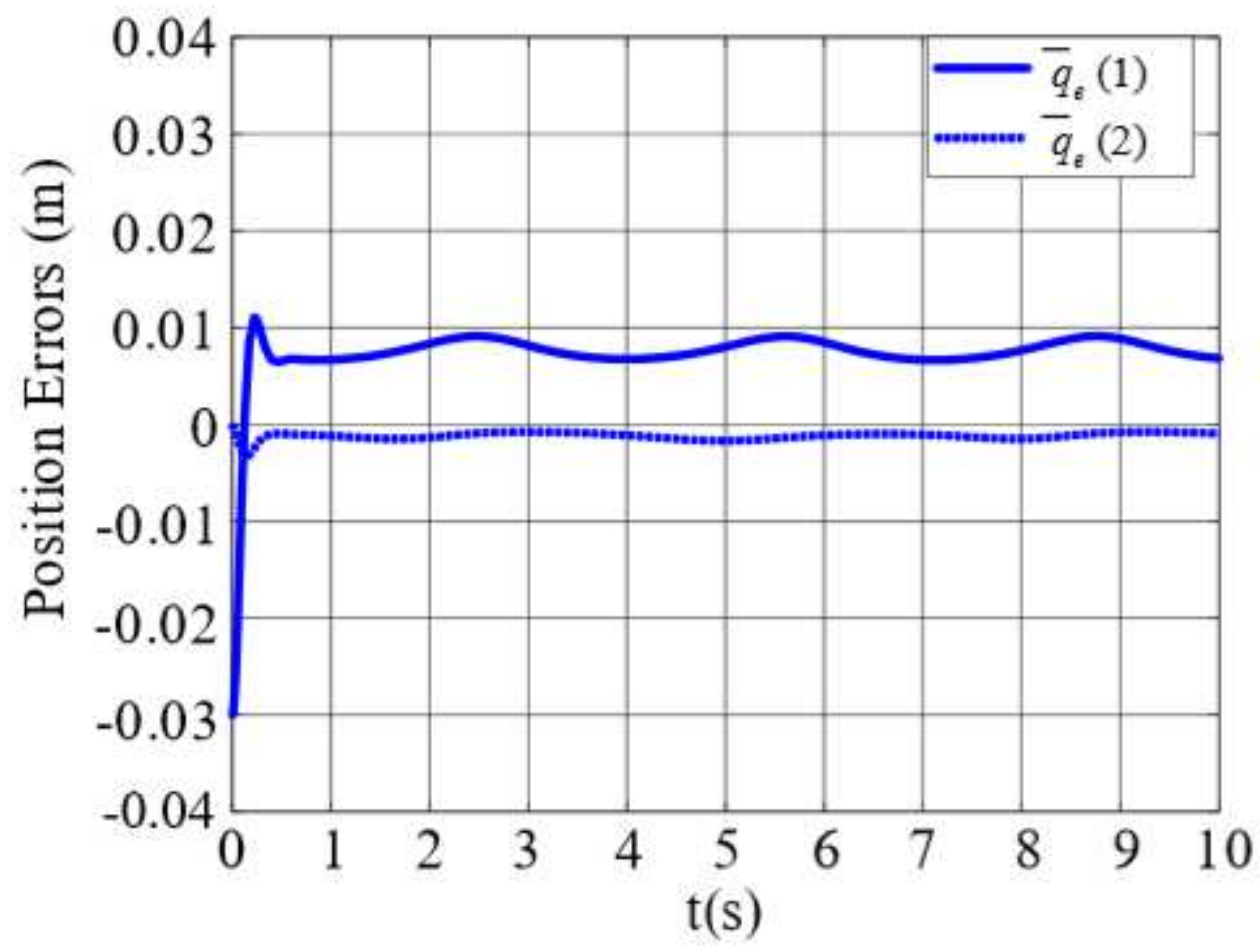

Figure 8 
Position errors trajectory of $\mathrm{EE}$

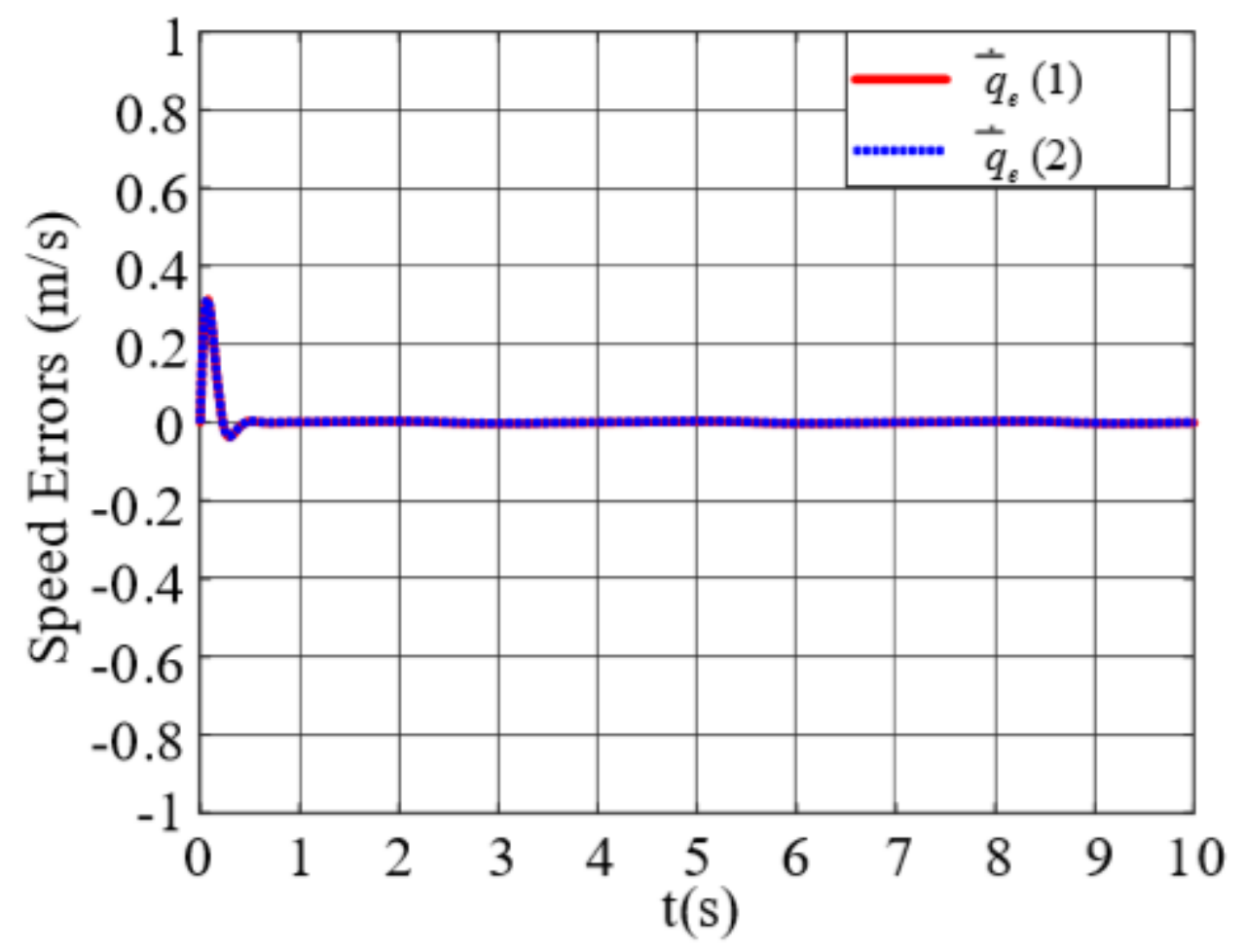

Figure 9

Speed errors trajectory of EE

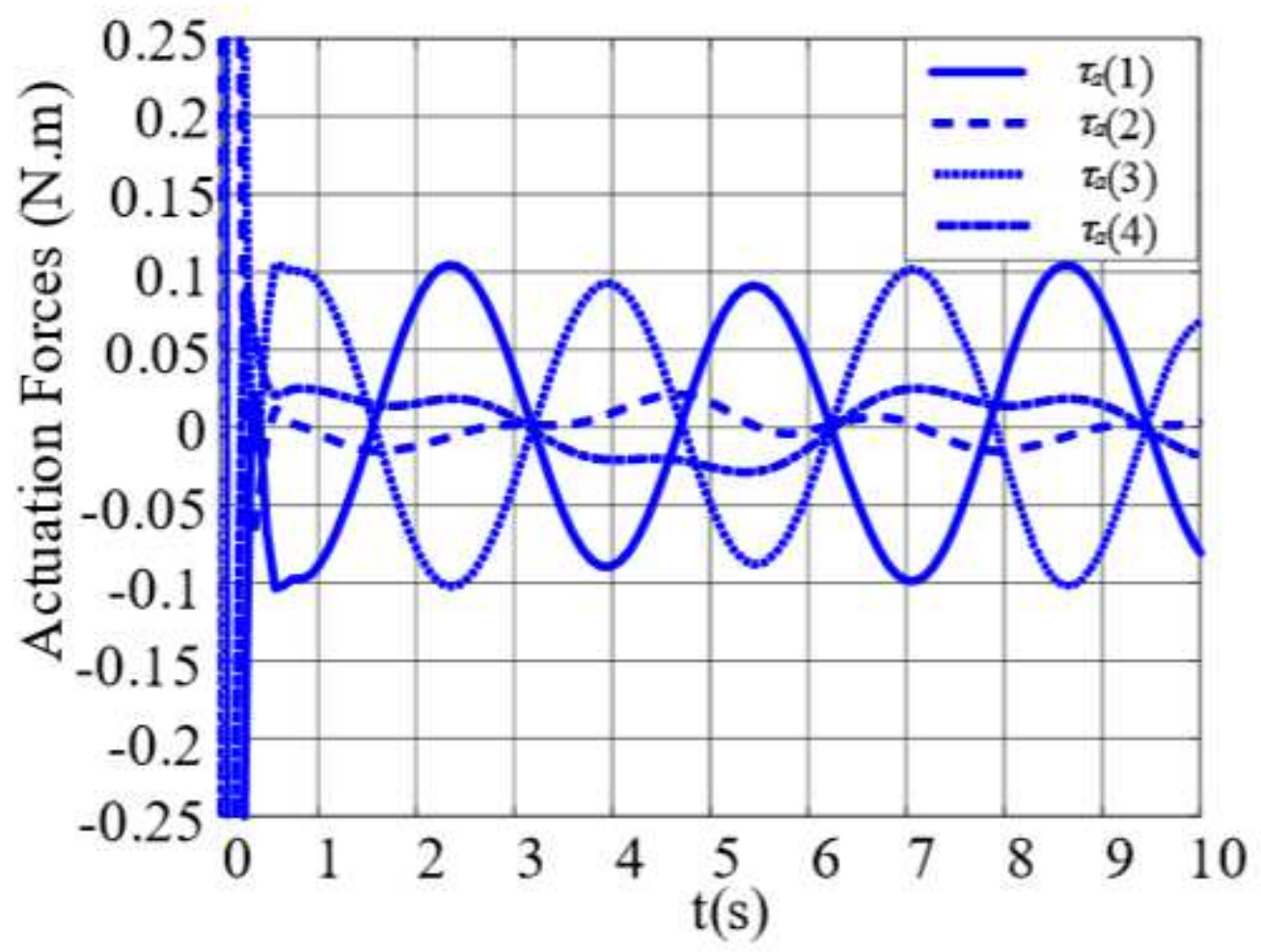

Figure 10 
Actuated moment trajectory of each actuated unit

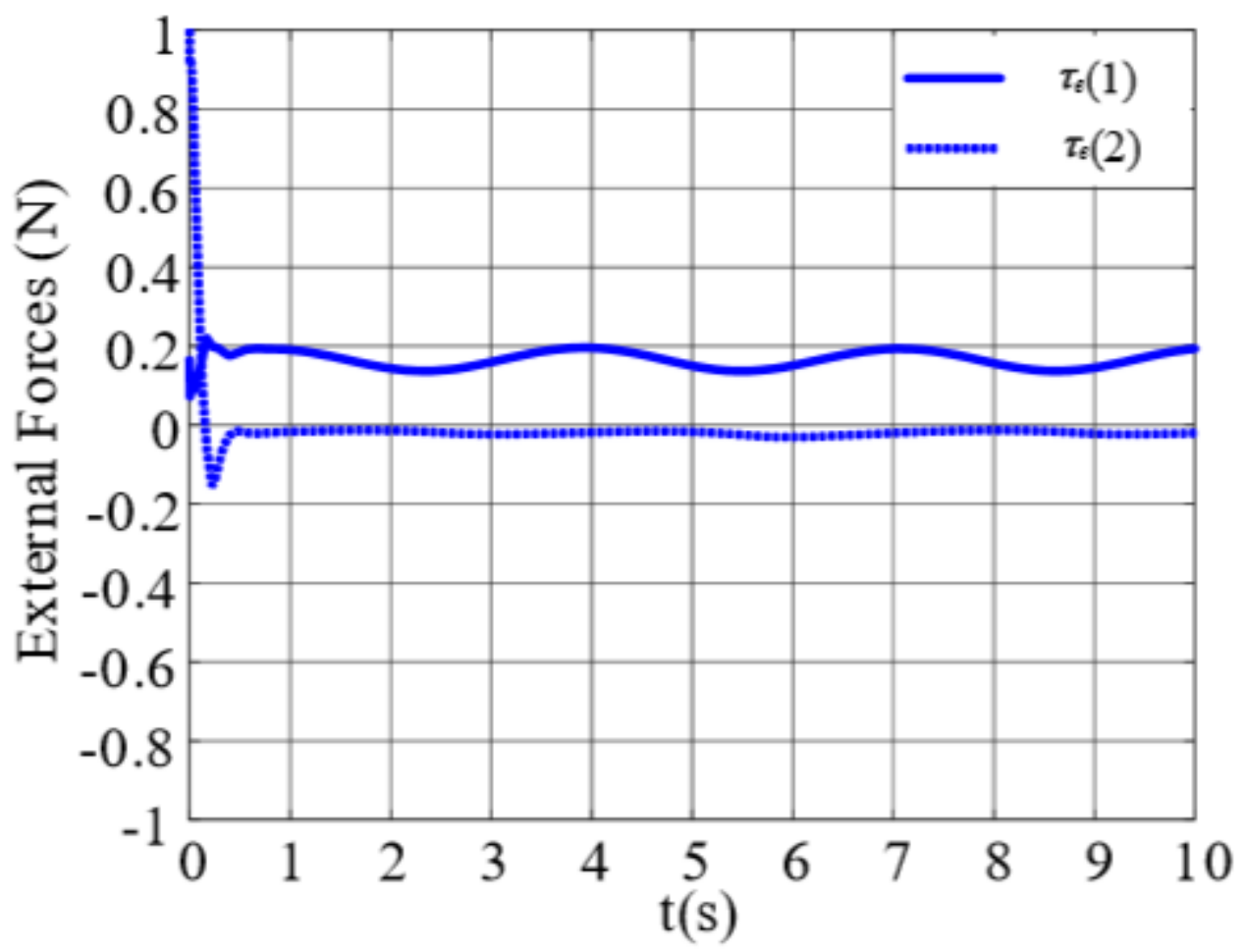

Figure 11

Interaction forces 